\title{
MATHEMATICAL ANALYSIS OF TEMPERATURE ACCELERATED DYNAMICS
}

\author{
DAVID ARISTOFF* AND TONY LELIÈVRE ${ }^{\dagger}$
}

\begin{abstract}
We give a mathematical framework for temperature accelerated dynamics (TAD), an algorithm proposed by M.R. Sørensen and A.F. Voter in [27] to efficiently generate metastable stochastic dynamics. Using the notion of quasistationary distributions, we propose some modifications to TAD. Then considering the modified algorithm in an idealized setting, we show how TAD can be made mathematically rigorous.
\end{abstract}

Key words. accelerated molecular dynamics, temperature accelerated dynamics, Langevin dynamics, stochastic dynamics, metastability, quasi-stationary distributions, kinetic Monte Carlo

AMS subject classifications. $82 \mathrm{C} 21,82 \mathrm{C} 80$

1. Introduction. Consider the stochastic dynamics $X_{t}$ on $\mathbb{R}^{d}$ satisfying

$$
d X_{t}=-\nabla V\left(X_{t}\right) d t+\sqrt{2 \beta^{-1}} d W_{t},
$$

called Brownian dynamics or overdamped Langevin dynamics. Here $V: \mathbb{R}^{d} \rightarrow \mathbb{R}$ is a smooth function, $\beta=\left(k_{B} T\right)^{-1}$ is a positive constant, and $W_{t}$ is a standard $d$ dimensional Brownian motion [23. The dynamics (1.1) is used to model the evolution of the position vector $X_{t}$ of $N$ particles (in which case $d=3 N$ ) in an energy landscape defined by the potential energy $V$. This is the so-called molecular dynamics. Typically this energy landscape has many metastable states, and in applications it is of interest to understand how $X_{t}$ moves between them. Temperature accelerated dynamics (TAD) is an algorithm for computing this metastable dynamics efficiently. (See 27. for the original algorithm, [19] for some modifications, and [25] for an overview of TAD and other similar methods for accelerating dynamics.)

Each metastable state corresponds to a basin of attraction $D$ for the gradient dynamics $d x / d t=-\nabla V(x)$ of a local minimum of the potential $V$. In TAD, temperature is raised to force $X_{t}$ to leave each basin more quickly. What would have happened at the original low temperature is then extrapolated. To generate metastable dynamics of $\left(X_{t}\right)_{t \geq 0}$ at low temperature, this procedure is repeated in each basin. This requires the assumptions:

(H1) $X_{t}$ immediately reaches local equilibrium upon entering a given basin $D$; and

(H2) An Arrhenius law may be used to extrapolate the exit event at low temperature.

The Arrhenius (or Eyring-Kramers) law states that, in the small temperature regime, the time it takes to transition between neighboring basins $D$ and $D^{\prime}$ is

$$
\nu^{-1} \exp \left[\frac{|\delta V|}{k_{B} T}\right],
$$

where $\delta V$ is the difference in potential energy between the local minimum in $D$ and the lowest saddle point along a path joining $D$ to $D^{\prime}$. Here $\nu$ is a constant (called a prefactor) depending on the eigenvalues of the Hessian of $V$ at the local minimum and at the saddle point, but not on the temperature. In practice the Arrhenius law is used when $k_{B} T \ll|\delta V|$. We refer to [2, 4, 11, 17] for details.

\footnotetext{
*Department of Mathematics, University of Minnesota

†CERMICS, École des Ponts ParisTech
} 
TAD is a very popular technique, in particular for applications in material sciences; see for example [1, 3, 5, 12, 18, 24, 28, 29, 30, 31, 32. In this article we provide a mathematical framework for TAD, and in particular a mathematical formalism for (H1)-(H2). Our analysis will actually concern a slightly modified version of TAD. In this modified version, which we call modified TAD, the dynamics is allowed to reach local equilibrium after entering a basin, thus circumventing assumption (H1). The assumption (H1) is closely related to the no recrossings assumption in transition state theory; in particular one can see the local equilibration steps (modifications (M1) and (M2) below) in modified TAD as a way to account for recrossings. We note that modified TAD can be used in practice and, since it does not require the assumption (H1), may reduce some of the numerical error in (the original) TAD.

To analyze modified TAD, we first make the notion of local equilibration precise by using quasistationary distributions, in the spirit of [15], and then we circumvent (H2) by introducing an idealized extrapolation procedure which is exact. The result, which we call idealized TAD, yields exact metastable dynamics; see Theorem 3.6 below. Idealized TAD is not a practical algorithm because it depends on quantities related to quasistationary distributions which cannot be efficiently computed. However, we show that idealized TAD agrees with modified TAD at low temperature. In particular we justify (H2) in modified TAD by showing that at low temperature, the extrapolation procedure of idealized TAD agrees with that of modified TAD (and of TAD), which is based on the Arrhenius law (1.2); see Theorem 4.1 below.

In this article, we focus on the overdamped Langevin dynamics (1.1) for simplicity. The algorithm is more commonly used in practice with the Langevin dynamics

$$
\left\{\begin{array}{l}
d q_{t}=M^{-1} p_{t} d t \\
d p_{t}=-\nabla V\left(q_{t}\right) d t-\gamma M^{-1} p_{t} d t+\sqrt{2 \gamma \beta^{-1}} d W_{t}
\end{array} .\right.
$$

The notion of quasistationary distributions still makes sense for the Langevin dynamics 22, so an extension of our analysis to that dynamics is in principle possible, though the mathematics there are much more difficult due to the degeneracy of the infinitesimal generator of (1.3). In particular, some results on the low temperature asymptotics of the principal eigenvalue and eigenvector for hypoelliptic diffusions are still missing.

The paper is organized as follows. In Section 2, we recall TAD and present modified TAD. In Section 3, we introduce idealized TAD and prove it is exact in terms of metastable dynamics. Finally, in Section 4, we show that idealized TAD and modified TAD are essentially equivalent in the low temperature regime. Our analysis in Section 4 is restricted to a one-dimensional setting. The extension of this to higher dimensions will be the purpose of another work.

Throughout the paper it will be convenient to refer to various objects related to the dynamics (1.1) at a high and low temperature, $\beta^{h i}$ and $\beta^{l o}$, as well as at a generic temperature, $\beta$. To do so, we use superscripts ${ }^{h i}$ and ${ }^{l o}$ to indicate that we are looking at the relevant object at $\beta=\beta^{h i}$ or $\beta=\beta^{l o}$, respectively. We drop the superscripts to consider objects at a generic temperature $\beta$.

2. TAD and modified TAD. Let $X_{t}^{\text {lo }}$ be a stochastic dynamics obeying (1.1) at a low temperature $\beta=\beta^{l o}$, and let $S: \mathbb{R}^{d} \rightarrow \mathbb{N}$ be a function which labels the basins of $V$. (So each basin $D$ has the form $S^{-1}(i)$ where $i \in \mathbb{N}$.) The goal of TAD is to efficiently estimate the metastable dynamics at low temperature; in other words:

- Efficiently generate a trajectory $\hat{S}(t)_{t \geq 0}$ which has approximately the same distribution as $S\left(X_{t}^{l o}\right)_{t \geq 0}$. 
The aim then is to get approximations of trajectories, including distributions of hitting times, time correlations, etc... and thus not only the evolution of the averages of some observables or averages of observables with respect to the invariant distribution.

At the heart of TAD is the problem of efficiently simulating an exit of $X_{t}^{\text {lo }}$ from a generic basin $D$, since the metastable dynamics are generated by essentially repeating this. To efficiently simulate an exit of $X_{t}^{l o}$ from $D$, temperature is raised so that $\beta^{h i}<\beta^{l o}$ and a corresponding high temperature dynamics $X_{t}^{h i}$ is evolved. The process $X_{t}^{h i}$ is allowed to search for various exit paths out of $D$ until a stopping time $T_{\text {stop}}$; each time $X_{t}^{h i}$ reaches $\partial D$ it is reflected back into $D$, the place and time of the attempted exit is recorded, and the Arrhenius law (1.2) is used to extrapolate a low temperature exit. After time $T_{\text {stop }}$ the fastest extrapolated low temperature exit is selected. This exit is considered an approximation of the first exit of $X_{t}^{l o}$ from $D$. The original algorithm is described in Section 2.1 below; a modified version is proposed in Section 2.2 below.

2.1. TAD. In the following, we let $D$ denote a generic basin. We let $x_{0}$ be the minimum of $V$ inside $D$, and we assume there are finitely many saddle points, $x_{i}$ $(i \geq 1)$, of $V$ on $\partial D$. The original TAD algorithm [27] for generating the approximate metastable dynamics $\hat{S}(t)$ is as follows:

Algorithm 2.1 (TAD). Let $X_{0}^{\text {hi }}$ be in the basin D, and start a low temperature simulation clock $T_{\text {tad }}$ at zero: $T_{\text {tad }}=0$. Then iterate on the visited basins the following:

1. Let $T_{\text {sim }}=0$ and $T_{\text {stop }}=\infty$. These are the simulation and stopping times for the high temperature exit search.

2. Evolve $X_{t}^{\text {hi }}$ at $\beta=\beta^{\text {hi }}$ starting at $t=T_{\text {sim }}$ until the first time after $T_{\text {sim }}$ at which it exits $D$. (Exits are detected by checking if the dynamics lands into another basin via gradient descent, i.e. the deterministic dynamics $d x / d t=$ $-\nabla V(x)$.) Call this time $T_{\text {sim }}+\tau$.

3. Associate a nearby saddle point, $x_{i}$, of $V$ on $\partial D$ to the place where $X_{t}^{\text {hi }}$ exited $D$. (This can be done by using, for example, the nudged elastic band method [14]; see below.)

4. Advance the high temperature simulation clock by $\tau: T_{\text {sim }}=T_{\text {sim }}+\tau$.

5. If an exit at $x_{i}$ has already been observed, go to Step 8. If an exit at $x_{i}$ has not yet been observed, set $T_{i}^{h i}=T_{\text {sim }}$ and extrapolate the high temperature exit time to low temperature using the formula:

$$
T_{i}^{l o}=T_{i}^{h i} e^{-\left(\beta^{h i}-\beta^{l o}\right)\left(V\left(x_{i}\right)-V\left(x_{0}\right)\right)} .
$$

This equation comes from the Arrhenius law (1.2) for exit rates in the low temperature regime; see the remarks below.

6. Update the smallest extrapolated exit time:

$$
T_{m i n}^{l o}=\min \left\{T_{m i n}^{l o}, T_{i}^{l o}\right\},
$$

and the (index of) the corresponding exit point:

$$
I_{\text {min }}^{l o}=i \quad \text { if } \quad T_{m i n}^{l o}=T_{i}^{l o} .
$$

7. Update $T_{\text {stop. }}$. The stopping time is chosen so that with confidence $1-\delta$, an extrapolated low temperature exit time smaller than $T_{\text {min }}^{\text {lo }}$ will not be observed. See equation (2.8) below for how this is done. 
8. If $T_{\text {sim }} \leq T_{\text {stop }}$, reflect $X_{t}^{\text {hi }}$ back into $D$ and go back to Step 2. Otherwise, proceed to Step 9.

9. Set

$$
\hat{S}(t)=S(D) \quad \text { for } \quad t \in\left[T_{t a d}, T_{t a d}+T_{m i n}^{l o}\right],
$$

and advance the low temperature simulation clock by $T_{\text {min }}^{\text {lo }}$ :

$$
T_{t a d}=T_{t a d}+T_{m i n}^{l o}
$$

10. Send $X_{t}^{h i}$ to the new basin, namely the neighboring basin of $D$ which is attained through the saddle point $x_{I_{\text {min }}^{\text {lo }}}$. Then, go back to Step 1, the domain $D$ now being the neighboring basin.

The nudged elastic band method [14] consists, starting from a trajectory leaving $D$, of computing by a gradient descent method the closest minimum energy path leaving $D$, with the end points of the trajectory being fixed. This minimum energy path necessarily leaves $D$ through a saddle point.

REMARK 2.2. When the overdamped Langevin dynamics leaves a basin near a saddle point, its first re-entrance into that basin is immediate. Thus, Algorithm 2.1 does not really make sense for overdamped Langevin dynamics. (With the Langevin dynamics (1.3), however, this difficulty does not arise.) In modified TAD, defined below, we will allow the dynamics to evolve away from the boundary of a basin after an exit event, thus circumventing this problem.

Below we comment on the equation (2.1) from which low temperature exit times are extrapolated, as well as the stopping time $T_{\text {stop }}$.

\section{- Low temperature extrapolation.}

The original TAD uses the following kinetic Monte Carlo (KMC) framework [33. For a given basin $D$, it is assumed that the time $\tilde{T}_{i}$ to exit through the saddle point $x_{i}$ of $V$ on $\partial D$ is exponentially distributed with rate $\kappa_{i}$ given by the Arrhenius law (1.2):

$$
\kappa_{i} \equiv \nu_{i} e^{-\beta\left(V\left(x_{i}\right)-V\left(x_{0}\right)\right)}
$$

where we recall $\nu_{i}$ is a temperature independent prefactor and $x_{0}$ is the minimum of $V$ in $D$. An exit event from $D$ at temperature $\beta$ is obtained by sampling independently the times $\tilde{T}_{i}$ for all the saddle points $x_{i}$ on $\partial D$, then selecting the smallest time and the corresponding saddle point.

In TAD, this KMC framework is used for both temperatures $\beta^{l o}$ and $\beta^{h i}$. That is, it is assumed that the high and low temperature exit times $\tilde{T}_{i}^{h i}$ and $\tilde{T}_{i}^{l o}$ through each saddle point $x_{i}$ satisfy:

$$
\begin{aligned}
& \mathbb{P}\left(\tilde{T}_{i}^{h i}>t\right)=e^{-\kappa_{i}^{h i} t} \\
& \mathbb{P}\left(\tilde{T}_{i}^{l o}>t\right)=e^{-\kappa_{i}^{l o} t}
\end{aligned}
$$

where

$$
\begin{aligned}
\kappa_{i}^{h i} & =\nu_{i} e^{-\beta^{h i}\left(V\left(x_{i}\right)-V\left(x_{0}\right)\right)} \\
\kappa_{i}^{l o} & =\nu_{i} e^{-\beta^{l o}\left(V\left(x_{i}\right)-V\left(x_{0}\right)\right)}
\end{aligned}
$$

Observe that then

$$
\tilde{T}_{i}^{h i} \frac{\kappa_{i}^{h i}}{\kappa_{i}^{l o}}=\tilde{T}_{i}^{h i} e^{-\left(\beta^{h i}-\beta^{l o}\right)\left(V\left(x_{i}\right)-V\left(x_{0}\right)\right)}
$$


has the same probability law as $\tilde{T}_{i}^{l o}$. This leads to the extrapolation formula (2.1).

The assumption of exponentially distributed exit times $T_{i}^{h i}$ and $T_{i}^{l o}$ is valid only if the dynamics at both temperatures immediately reach local equilibrium upon entering a basin; see (H1) and Theorem 3.1 below. In modified TAD, described below, we circumvent this immediate equilibration assumption by allowing the dynamics at both temperatures to reach local equilibrium. In particular, in modified TAD the low temperature assumption is no longer needed to get exponential exit distributions as in (2.3). On the other hand, to get the rate constants in (2.4) - and by extension the extrapolation rule (2.1); see (H2) - a low temperature assumption is required. We will justify both (2.3) and (2.4) in the context of modified TAD. More precisely we show that (2.3) will be valid at any temperature, while a low temperature assumption is needed to justify (2.4). Note that, inspecting equation (2.4), the low temperature assumption will be required for both temperatures used in TAD - so $1 / \beta^{h i}$ will be small in an absolute sense, but large compared to $1 / \beta^{l o}$.

\section{- Stopping time.}

The stopping time $T_{\text {stop }}$ is chosen so that if the high temperature exit search is stopped at time $T_{\text {stop }}$, then with probability $1-\delta$, the smallest extrapolated low temperature exit time will be correct. Here $\delta$ is a user-specified parameter. To obtain a formula for the stopping time $T_{\text {stop }}$ it is assumed that, in addition to $(\mathrm{H} 1)-(\mathrm{H} 2)$ :

(H3) There is a minimum, $\nu_{m i n}$, to all the prefactors in equation (2.4):

$$
\forall i \in\{1, \ldots k\}, \nu_{i} \geq \nu_{\min }
$$

where $k$ denotes the number of saddle points on $\partial D$.

Let us now explain how this assumption is used to determine $T_{\text {stop }}$. Let $T$ be a deterministic time. If a high temperature first exit time through $x_{i}, T_{i}^{h i}>T$, extrapolates to a low temperature time less than $T_{\text {min }}^{l o}$, then from (2.1),

$$
V\left(x_{i}\right)-V\left(x_{0}\right) \leq \frac{\log \left(T_{m i n}^{l o} / T\right)}{\beta^{l o}-\beta^{h i}}
$$

and so

$$
\kappa_{i}^{h i}=\nu_{i} e^{-\beta^{h i}\left(V\left(x_{i}\right)-V\left(x_{0}\right)\right)} \geq \nu_{\min } \exp \left(\frac{\beta^{h i} \log \left(T_{\min }^{l o} / T\right)}{\beta^{h i}-\beta^{l o}}\right) .
$$

In TAD it is required that this event has a low probability $\delta$ of occurring, that is,

$$
\mathbb{P}\left(T_{i}^{h i}>T\right)=e^{-\kappa_{i}^{h i} T}<\delta .
$$

Using (2.5) in (2.6), one sees that it suffices that

$$
\exp \left[-\nu_{\min } \exp \left(\frac{\beta^{h i} \log \left(T_{\min }^{l o} / T\right)}{\beta^{h i}-\beta^{l o}}\right) T\right]<\delta \text {. }
$$

Solving this inequality for $T$, one obtains

$$
T>\frac{\log (1 / \delta)}{\nu_{\min }}\left(\frac{\nu_{\min } T_{\min }^{l o}}{\log (1 / \delta)}\right)^{\beta^{h i} / \beta^{l o}} .
$$


The stopping time $T_{\text {stop }}$ is then chosen to be the right hand side of the above:

$$
T_{\text {stop }} \equiv \frac{\log (1 / \delta)}{\nu_{\min }}\left(\frac{\nu_{\min } T_{\min }^{l o}}{\log (1 / \delta)}\right)^{\beta^{h i} / \beta^{l o}} .
$$

(It is calculated using the current value of $T_{m i n}^{l o}$.) The above calculation shows that at simulation time $T_{\text {stop }}$, with probability at least $1-\delta, T_{\min }^{l o}$ is the same as the smallest extrapolated low temperature exit time which would have been observed with no stopping criterion.

For TAD to be practical, the stopping time $T_{\text {stop }}$ must be (on average) smaller than the exit times at low temperature. The stopping time of course depends on the choice of $\nu_{\min }$ and $\delta$. In practice a reasonable value for $\nu_{\min }$ may be known a priori 27] or obtained by a crude approximation 25. For a given $\delta$, if too large a value of $\nu_{\min }$ is used, the low temperature extrapolated times may be incorrect with probability greater than $\delta$. On the other hand, if the value of $\nu_{\min }$ is too small, then the extrapolated times will be correct with probability $1-\delta$, but computational efficiency will be compromised. The usefulness of TAD comes from the fact that, in practice, $\nu_{\min }$ and $\delta$ can often be chosen such that the correct low temperature exit event is found by time $T_{\text {stop }}$ with large probability $1-\delta$, and $T_{\text {stop }}$ is on average much smaller than the exit times which would be expected at low temperature. In practical applications, TAD has provided simulation time scale boosts of up to $10^{9}[28$.

REMARK 2.3. One alternative to TAD is a brute force saddle point search method, in which one evolves the system at a high temperature $\beta^{\text {hi }}$ to locate saddle points of $V$ on $\partial D$. (There are other popular techniques in the literature to locate saddle points, many of which do not use high temperature or dynamics; see for example [21].) Once one is confident that all the physically relevant saddle points are found, the times $\tilde{T}_{i}^{l o}$ to exit through each $x_{i}$ at low temperature can be directly sampled from exponential distributions with parameters $\kappa_{i}$ as in (2.2), using $\beta \equiv \beta^{\text {lo }}$. (Estimates are available for the $\nu_{i}$ at low temperature; they depend on the values of $V$ and the Hessian matrix of $V$ at $x_{i}$ and $x_{0}$. See for example [4].)

The advantage of TAD over a brute force saddle point search method is that in $T A D$, there is a well-defined stopping criterion for the saddle point search at temperature $\beta^{\text {hi }}$, in the sense that the saddle point corresponding to the correct exit event at temperature $\beta^{\text {lo }}$ will be obtained with a user-specified probability. In particular, TAD does not require all the saddle points to be found.

2.2. Modified TAD. Below we consider some modifications, (M1)-(M3), to TAD, calling the result modified TAD. The main modifications, (M1)-(M2) below, will ensure that the exponential rates assumed in TAD are justified. We also introduce a different stopping time, (M3). (See the discussion below Algorithm 2.5) We note that some of these features are currently being used by practitioners of TAD [35]. Here are the three modifications:

(M1) We include a decorrelation step in which an underlying low temperature dynamics $\left(X_{t}^{l o}\right)_{t \geq 0}$ finds local equilibrium in some basin $D$ before we start searching for exit pathways at high temperature;

(M2) Before searching for exit pathways out of $D$, we sample local equilibrium at high temperature in the current basin $D$, without advancing any clock time;

(M3) We replace the stopping time (2.8) with

$$
T_{\text {stop }}=T_{\text {min }}^{l o} / C,
$$


where $C$ is a lower bound of the minimum of $e^{-\left(\beta^{h i}-\beta^{l o}\right)\left(V\left(x_{i}\right)-V\left(x_{0}\right)\right)}$ over all the saddle points, $x_{i}$, of $V$ on $\partial D$.

REMARK 2.4. In (M3) above we are assuming some a priori knowledge of the system, in particular a lower bound of the energy barriers $V\left(x_{i}\right)-V\left(x_{0}\right), i \in\{1, \ldots k\}$. Such a lower bound will not be known in every situation, but in some cases, practitioners can obtain such a bound, see for example [19]. See also the discussion in the section "Stopping time" below.

The modified algorithm is as follows; for the reader's convenience we have boxed off the steps of modified TAD which are different from TAD.

Algorithm 2.5 (Modified TAD). Let $X_{0}^{\text {lo }}$ be in the basin D, set a low temperature simulation clock $T_{\text {tad }}$ to zero: $T_{\text {tad }}=0$, and choose a (basin-dependent) decorrelation time $T_{\text {corr }}>0$. Then iterate on the visited basins the following:

\section{Decorrelation step:}

1. Starting at time $t=T_{\text {tad }}$, evolve $X_{t}^{\text {lo }}$ at temperature $\beta=\beta^{\text {lo }}$ according to (1.1) in the current basin $D$.

2. If $X_{t}^{\text {lo }}$ exits $D$ at a time $T_{t a d}+\tau<T_{\text {tad }}+T_{\text {corr }}$, then set

$$
\hat{S}(t)=S(D), \quad t \in\left[T_{t a d}, T_{t a d}+\tau\right],
$$

advance the low temperature clock by $\tau: T_{t a d}=T_{t a d}+\tau$, then go back to Step 1, where $D$ is now the new basin. Otherwise, set

$$
\hat{S}(t)=S(D), \quad t \in\left[T_{t a d}, T_{t a d}+T_{\text {corr }}\right],
$$

advance the low temperature clock by $T_{\text {corr }}: T_{t a d}=T_{t a d}+T_{\text {corr }}$, and initialize the exit step by setting $T_{\text {sim }}=0$ and $T_{\text {stop }}=\infty$. Then proceed to the exit step.

\section{Exit step:}

1. Let $X_{T_{s i m}}^{h i}$ be a sample of the dynamics (1.1) in local equilibrium in $D$ at temperature $\beta=\beta^{\text {hi }}$. See the remarks below for how this sampling is done. None of the clocks are advanced in this step.

2. Evolve $X_{t}^{\text {hi }}$ at $\beta=\beta^{\text {hi }}$ starting at $t=T_{\text {sim }}$ until the first time after $T_{\text {sim }}$ at which it exits D. Call this time $T_{\text {sim }}+\tau$.

3. Using the nudged elastic band method, associate a nearby saddle point, $x_{i}$, of $V$ on $\partial D$ to the place where $X_{t}^{\text {hi }}$ exited $D$.

4. Advance the simulation clock by $\tau: T_{\text {sim }}=T_{\text {sim }}+\tau$.

5. If an exit at $x_{i}$ has already been observed, go to Step 8. If an exit at $x_{i}$ has not yet been observed, set $T_{i}^{h i}=T_{\text {sim }}$ and

$$
T_{i}^{l o}=T_{i}^{h i} e^{-\left(\beta^{h i}-\beta^{l o}\right)\left(V\left(x_{i}\right)-V\left(x_{0}\right)\right)} .
$$

6. Update the lowest extrapolated exit time:

$$
T_{m i n}^{l o}=\min \left\{T_{m i n}^{l o}, T_{i}^{l o}\right\},
$$

and the (index of) the corresponding exit point:

$$
I_{\text {min }}^{l o}=i \quad \text { if } \quad T_{\text {min }}^{l o}=T_{i}^{l o} .
$$


7. Update $T_{\text {stop }}$ :

$$
T_{\text {stop }}=T_{m i n}^{l o} / C,
$$

where $C$ is a lower bound of the minimum of $e^{-\left(\beta^{h i}-\beta^{l o}\right)\left(V\left(x_{i}\right)-V\left(x_{0}\right)\right)}$ over all the saddle points, $x_{i}$, of $V$ on $\partial D$.

8. If $T_{\text {sim }} \leq T_{\text {stop }}$, go back to Step 1 of the exit step; otherwise, proceed to Step 9.

9. Set

$$
\hat{S}(t)=S(D) \quad \text { for } \quad t \in\left[T_{t a d}, T_{t a d}+T_{m i n}^{l o}\right],
$$

and advance the low temperature simulation clock by $T_{m i n}^{l o}$ :

$$
T_{t a d}=T_{t a d}+T_{m i n}^{l o}
$$

10. Set $X_{T_{t a d}}^{l o}=X_{T_{I}^{h i}}^{h i}$ where $I \equiv I_{m i n}^{l o}$. Then go back to the decorrelation step, the domain $D$ now being the neighboring basin, namely the one obtained by exiting through $X_{T_{I}^{h i}}^{h i}$.

- Local equilibrium in $D$ : (M1) and (M2).

We introduce the decorrelation step - see (M1) - in order to ensure that the low temperature dynamics reaches local equilibrium in $D$. Indeed, for sufficiently large $T_{\text {corr }}$ the low temperature dynamics reaches local equilibrium in some basin. The convergence to local equilibrium will be made precise in Section 3 using the notion of the quasistationary distribution. See also [26, 15], in particular for a discussion of the choice of $T_{\text {corr }}$. Local equilibrium will in general be reached at different times in different basins, so we allow $T_{\text {corr }}$ to be basin dependent. We note that a similar decorrelation step is used in another accelerated dynamics proposed by A.F. Voter, the Parallel Replica Dynamics 34. The decorrelation step accounts for barrier recrossing events: the dynamics is allowed to evolve exactly at low temperature after the exit step, capturing any possible barrier recrossings, until local equilibrium is reached in one of the basins.

The counterpart of the addition of this decorrelation step is that, from (M2), in the exit step we also start the high temperature dynamics from local equilibrium in the current basin $D$. A similar step is actually being used by current practitioners of TAD [35], though this step is not mentioned in the original algorithm [27. To sample local equilibrium in $D$, one can for example take the end position of a a sufficiently long trajectory of (1.1) which does not exit $D$. See [15, 26 for some algorithms to efficiently sample local equilibrium; we remark that this is expected to become more computationally demanding as temperature increases.

To extrapolate the exit event at low temperature from the exit events at high temperature, we need the dynamics at both temperatures to be in local equilibrium. We note that the changes (M1)-(M2) in modified TAD are actually a practical way to get rid of the error associated with the assumption (H1) in TAD.

- Stopping time: (M3).

In (M3) we introduce a stopping $T_{\text {stop }}$ such that, with probability 1 , the shortest extrapolated low temperature exit time is found by time $T_{\text {stop }}$. (Recall that with the stopping time of TAD, we have only a confidence level $1-\delta$.) 
Note that for the stopping time $T_{\text {stop }}$ to be implemented in (2.11), we need some a priori knowledge about energy barriers, in particular a lower bound $E_{\min }>0$ for all the differences $V\left(x_{i}\right)-V\left(x_{0}\right)$, where $x_{i}$ ranges over the saddle points on the boundary of a given basin:

(H3') There is a minimum, $E_{m i n}$, to all the energy barriers:

$$
\forall i \in\{1, \ldots k\}, V\left(x_{i}\right)-V\left(x_{0}\right) \geq E_{\min } .
$$

If a lower bound $E_{\min }$ is known, then we can choose $C$ accordingly so that in equation (2.11) we obtain

$$
T_{\text {stop }}=T_{m i n}^{l o} e^{\left(\beta^{h i}-\beta^{l o}\right) E_{m i n}} .
$$

A simple computation then shows that under assumption (H3'), any high temperature exit time occurring after $T_{\text {stop }}$ cannot extrapolate to a low temperature exit time smaller than $T_{\text {min }}^{l o}$. To see that (2.12) leads to an efficient algorithm, recall that TAD is expected to be correct only in the regime where $\beta^{h i} \gg E_{\min }$, which since $\beta^{h i} \ll \beta^{l o}$ means the exponential in (2.12) should be very small.

As the computational savings of TAD comes from the fact that the simulation time of the exit step, namely $T_{\text {stop }}$, is much smaller than the exit time that would have been observed at low temperature, the choice of stopping time in TAD is of critical importance. Both of the stopping times (2.8) and (2.9) are used in practice; see [19] for a presentation of TAD with the stopping formula (2.9), and [20] for an application. The original stopping time (2.8) requires a lower bound for the prefactors in the Arrhenius law (2.4) (see assumption (H3) above, in the remarks following Algorithm 2.1). The stopping time (2.9) requires an assumption on the minimum energy barriers; see assumption (H3') above. The formula (2.9) may be preferable in case minimum energy barriers are known, since it is known to scale better with system size than (2.8). The formula (2.8) is advantageous if minimum energy barriers are unknown but a reasonable lower bound for the minimum prefactor $\nu_{\min }$ is available.

We have chosen the stopping time (2.9) instead of (2.8) mostly for mathematical convenience - in particular so that in our Section 3 analysis we do not have the error $\delta$ associated with (2.8). A similar analysis can be done under assumption (H3) with the stopping time (2.8), modulo the error $\delta$.

We comment that modified TAD is an algorithm which can be implemented in practice, and which circumvents the error in the original TAD arising from the assumption (H1).

3. Idealized TAD and mathematical analysis. In this section we show that under certain idealizing assumptions, namely (I1)-(I3) and (A1) below, modified TAD is exact in the sense that the simulated metastable dynamics $\hat{S}(t)_{t \geq 0}$ has the same law as the true low temperature metastable dynamics $S\left(X_{t}^{l o}\right)_{t \geq 0}$. We call this idealization of modified TAD idealized TAD. Our analysis will show that idealized TAD and modified TAD agree in the limit $\beta^{h i}, \beta^{l o} \rightarrow \infty$ and $T_{\text {corr }} \rightarrow \infty$. Since idealized TAD is exact, it follows that modified TAD is exact in the limit $\beta^{h i}, \beta^{l o} \rightarrow \infty$ and $T_{\text {corr }} \rightarrow \infty$.

In idealized TAD, we assume that at the end of the decorrelation step and at the start of the exit step of modified TAD, we are in exact local equilibrium; see (A1) 


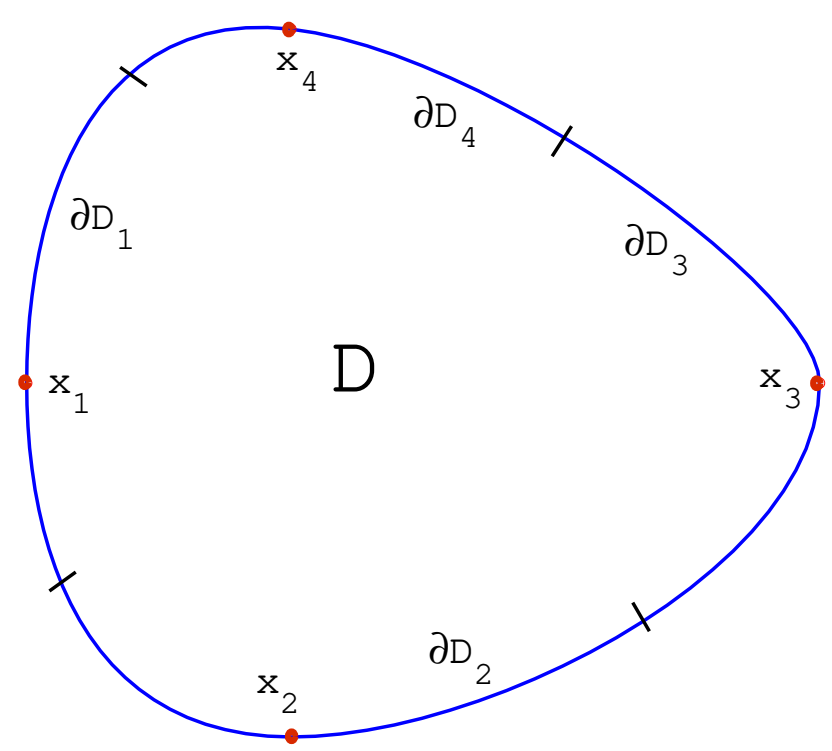

FIG. 3.1. The domain $D$ with boundary partitioned into $\partial D_{1}, \ldots, \partial D_{4}$ (here $\left.k=4\right)$ by the black line segments. $V$ has exactly one saddle point in each $\partial D_{i}$, located at $x_{i}$.

and (I1). We formalize this using the notion of quasistationary distributions, defined below. We also assume that the way in which we exit near a given saddle point $x_{i}$ in the exit step does not affect the metastable dynamics in the decorrelation step; see (I2). The remaining idealization, whose relation to modified TAD is maybe not so clear at first sight, is to replace the exponential $\exp \left[-\left(\beta^{h i}-\beta^{l o}\right)\left(V\left(x_{i}\right)-V\left(x_{0}\right)\right)\right]$ of (2.1) with a certain quantity $\Theta_{i}$ depending on the flux of the quasistationary distribution across $\partial D$; see (I3). In Section 4 we justify this by showing that the two agree asymptotically as $\beta^{\text {hi }}, \beta^{l o} \rightarrow \infty$ in a one-dimensional setting.

3.1. Notation and quasistationary distribution. Here and throughout, $D$ is an (open) domain with $C^{2}$ boundary $\partial D$ and $X_{t}^{x}$ is a stochastic process evolving according to (1.1) starting at $X_{0}^{x}=x$ (we suppress the superscript where it is not needed). We write $\mathbb{P}(\cdot)$ and $\mathbb{E}[\cdot]$ for various probabilities and expectations, the meaning of which will be clear from context. We write $Y \sim \mu$ for a random variable sampled from the probability measure $\mu$ and $Y \sim \mathcal{E}(\alpha)$ for an exponentially distributed random variable with parameter $\alpha$.

Recalling the notation of Section 2, we assume that $\partial D$ is partitioned into $k$ (Lebesgue measurable) subsets $\partial D_{i}$ containing the saddle points $x_{i}$ of $V, i=1, \ldots, k$ (see Fig 3.1):

$$
\partial D=\cup_{i=1}^{k} \partial D_{i} \quad \text { and } \quad \partial D_{i} \cap \partial D_{j}=\emptyset \text { if } i \neq j .
$$

We assume that any exit through $\partial D_{i}$ is associated to the saddle point $x_{i}$ in Step 3 of TAD. In other words, $\partial D_{i}$ corresponds to the basin of attraction of the saddle point $x_{i}$ for the nudged elastic band method.

Essential to the analysis below will be the notion of quasistationary distribution, which we define below, recalling some facts which will be needed in our analysis. 
Consider the infinitesimal generator of (1.1):

$$
L=-\nabla V \cdot \nabla+\beta^{-1} \Delta,
$$

and let $(u,-\lambda)$ be the principal eigenvector/eigenvalue pair for $L$ with homogeneous Dirichlet (absorbing) boundary conditions on $\partial D$ :

$$
\left\{\begin{aligned}
L u & =-\lambda u \text { in } D \\
u & =0 \text { on } \partial D .
\end{aligned}\right.
$$

It is known (see [15]) that $u$ is signed and $\lambda>0$; we choose $u>0$ and for the moment do not specify a normalization. Define a probability measure $\nu$ on $D$ by

$$
d \nu=\frac{u(x) e^{-\beta V(x)} d x}{\int_{D} u(x) e^{-\beta V(x)} d x} .
$$

The measure $\nu$ is called the quasistationary distribution (QSD) on $D$; the name comes from the fact that $\nu$ has the following property: for $\left(X_{t}\right)_{t \geq 0}$ a solution to (1.1), starting from any distribution with support in $D$,

$$
\nu(A)=\lim _{t \rightarrow \infty} \mathbb{P}\left(X_{t} \in A \mid X_{s} \in D, 0 \leq s \leq t\right) \quad \text { for any measurable set } A \subset D .
$$

The following is proved in [15], and will be essential for our results:

TheOREm 3.1. Let $X_{t}$ be a solution to (1.1) with $X_{0} \sim \nu$, and let

$$
\tau=\inf \left\{t>0: X_{t} \notin D\right\}
$$

Then: (i) $\tau \sim \mathcal{E}(\lambda)$ and (ii) $\tau$ and $X_{\tau}$ are independent. We will also need the following formula from 15 for the exit point distribution:

TheOrem 3.2. Let $X_{t}$ and $\tau$ be as in Theorem 3.1, and let $\sigma_{\partial D}$ be Lebesgue measure on $\partial D$. The measure $\rho$ on $\partial D$ defined by

$$
d \rho=-\frac{\partial_{n}\left(u(x) e^{-\beta V(x)}\right) d \sigma_{\partial D}}{\beta \lambda \int_{D} u(x) e^{-\beta V(x)} d x}
$$

is a probability measure, and for any measurable $A \subset \partial D$,

$$
\mathbb{P}\left(X_{\tau} \in A\right)=\rho(A) .
$$

As a corollary of these two results we have the following, which will be central to our analysis:

Corollary 3.3. Let $X_{t}$, $\tau$ and $\rho$ be as in Theorems 3.1]3.2, and define

$$
p_{i}=\rho\left(\partial D_{i}\right)
$$

to be the exit probability through $\partial D_{i}$. Let $I$ be the discrete random variable defined by: for $i=1, \ldots, k$,

$$
I=i \text { if and only if } X_{\tau} \in \partial D_{i} .
$$

Then (i) $\tau \sim \mathcal{E}(\lambda)$, (ii) $\mathbb{P}(I=i)=p_{i}$, and (iii) $\tau$ and $I$ are independent. 
Throughout we omit the dependence of $\lambda, \nu$, and $\rho$ on the basin $D$; it should be understood from context.

REMARK 3.4. We assume that $D$ has $C^{2}$ boundary so that standard elliptic regularity results and trace theorems give a meaning to the formula (3.4) used to define $\rho$ in Theorem 3.2. For basins of attraction this assumption will not be satisfied, as the basins will have "corners". This is actually a minor technical point. The probability measure $\rho$ can be defined for any Lipschitz domain $D$ using the following two steps: first, $\rho$ can be defined in $H^{-1 / 2}(\partial \Omega)$ using the definition (equivalent to (3.4) ): for any $v \in H^{1 / 2}(\partial D)$

$$
\langle v, d \rho\rangle=\frac{\int_{D}\left(-\beta^{-1} \nabla w \cdot \nabla u+\lambda w u\right) \exp (-\beta V)}{\lambda \int_{D} u \exp (-\beta V)}
$$

where $w \in H^{1}(D)$ is any lifting of $v\left(\left.w\right|_{\partial D}=v\right)$. Second, it is easy to check that $\rho$ actually defines a non-negative distribution on $\partial D$, for example by using as a lifting the solution to

$$
\left\{\begin{aligned}
L w & =0 \text { in } D, \\
w & =v \text { on } \partial D,
\end{aligned}\right.
$$

since, by the maximum principle, $w \geq 0$, and then, $\langle v, d \rho\rangle=\frac{\int_{D} \lambda w u \exp (-\beta V)}{\lambda \int_{D} u \exp (-\beta V)}$. One finally concludes using a Riesz representation theorem due to Schwartz: any nonnegative distribution with total mass one defines a probability measure.

3.2. Idealized TAD. In this section we consider an idealized version of modified TAD, which we call idealized TAD. The idealizations, (I1)-(I3) below, are introduced so that the algorithm can be rigorously analyzed using the mathematical formalisms in Section 3.1

(I1) At the start of the exit step, the high temperature dynamics is initially distributed according to the QSD in $D: X_{T_{s i m}}^{h i} \sim \nu^{h i}$;

(I2) At the end of the exit step, the extrapolated low temperature exit point $X_{T_{t a d}}^{l o}$ is sampled exactly from the conditional exit point distribution in $\partial D_{I_{\text {min }}^{l o}}$ at low temperature:

$$
\left.X_{T_{\text {tad }}}^{l o} \sim\left[\rho^{l o}\left(\partial D_{I_{\text {min }}^{l o}}\right)\right]^{-1} \rho^{l o}\right|_{\partial D_{I_{m i n}^{l o}}}
$$

(I3) In the exit step, the quantity

$$
e^{-\left(\beta^{h i}-\beta^{l o}\right)\left(V\left(x_{i}\right)-V\left(x_{0}\right)\right)}
$$

is everywhere replaced by

$$
\Theta_{i} \equiv \frac{\lambda^{h i} p_{i}^{h i}}{\lambda^{l o} p_{i}^{l o}}
$$

where, as in (3.5), $p_{i}^{l o}=\rho^{l o}\left(\partial D_{i}\right)$ and $p_{i}^{h i}=\rho^{h i}\left(\partial D_{i}\right)$. Thus, the extrapolation equation (2.1) is replaced by

$$
T_{i}^{l o}=T_{i}^{h i} \Theta_{i}
$$

and the formula for updating $T_{\text {stop }}$ is:

$$
T_{\text {stop }}=T_{\text {min }}^{l o} / C
$$

where $C$ is chosen so that $C \leq \min _{1 \leq i \leq k} \Theta_{i}$. 
We state idealized TAD below as an "algorithm", even though it is not practical: in general we cannot exactly sample $\nu^{h i}$ or the exit distributions $\left.\left[\rho^{l o}\left(\partial D_{i}^{l o}\right)\right]^{-1} \rho^{l o}\right|_{\partial D_{i}^{l o}}$, and the quantities $\Theta_{i}$ are not known in practice. (See the discussion below Algorithm [3.5])

For the reader's convenience we put in boxes those steps of idealized TAD which are different from modified TAD.

Algorithm 3.5 (Idealized TAD). Let $X_{0}^{\text {lo }}$ be in the basin D, set the low temperature clock time to zero: $T_{\text {tad }}=0$, let $T_{\text {corr }}>0$ be a (basin-dependent) decorrelation time, and iterate on the visited basins the following:

Decorrelation step:

1. Starting at time $t=T_{\text {tad }}$, evolve $X_{t}^{\text {lo }}$ at temperature $\beta=\beta^{\text {lo }}$ according to (1.1) in the current basin $D$.

2. If $X_{t}^{\text {lo }}$ exits $D$ at a time $T_{t a d}+\tau<T_{t a d}+T_{\text {corr }}$, then set

$$
\hat{S}(t)=S(D), \quad t \in\left[T_{t a d}, T_{t a d}+\tau\right],
$$

advance the low temperature clock by $\tau: T_{\text {tad }}=T_{\text {tad }}+\tau$, then go back to Step 1, where $D$ is now the new basin. Otherwise, set

$$
\hat{S}(t)=S(D), \quad t \in\left[T_{t a d}, T_{t a d}+T_{\text {corr }}\right],
$$

advance the low temperature clock by $T_{\text {corr }}: T_{\text {tad }}=T_{\text {tad }}+T_{\text {corr }}$, and initialize the exit step by setting $T_{\text {sim }}=0$ and $T_{\text {stop }}=\infty$. Then proceed to the exit step.

Exit step:

1. Sample $X_{T_{s i m}}^{h i}$ from the $Q S D$ at high temperature in $D: X_{T_{s i m}}^{h i} \sim \nu^{h i}$.

2. Evolve $X_{t}^{\text {hi }}$ at $\beta=\beta^{\text {hi }}$ starting at $t=T_{\text {sim }}$ until the first time after $T_{\text {sim }}$ at which it exits D. Call this time $T_{\text {sim }}+\tau$.

3. Record the set $\partial D_{i}$ through which $X_{t}^{h i}$ exited $D$.

4. Advance the simulation clock by $\tau: T_{\text {sim }}=T_{\text {sim }}+\tau$.

5. If an exit through $\partial D_{i}$ has already been observed, go to Step 8 . If an exit through $\partial D_{i}$ has not yet been observed, set $T_{i}^{h i}=T_{\text {sim }}$ and:

$$
T_{i}^{l o}=T_{i}^{h i} \Theta_{i}, \quad \Theta_{i} \equiv \frac{\lambda^{h i} p_{i}^{h i}}{\lambda^{l o} p_{i}^{l o}} .
$$

6. Update the lowest extrapolated exit time and corresponding exit spot:

$$
\begin{aligned}
& T_{m i n}^{l o}=\min \left\{T_{m i n}^{l o}, T_{i}^{l o}\right\} \\
& I_{\text {min }}^{l o}=i \quad \text { if } \quad T_{\text {min }}^{l o}=T_{i}^{l o} .
\end{aligned}
$$

7. Update $T_{\text {stop }}$ :

$$
T_{\text {stop }}=T_{\text {min }}^{l o} / C, \quad C \leq \min _{1 \leq i \leq k} \Theta_{i} .
$$

8. If $T_{\text {sim }} \leq T_{\text {stop }}$, go back to Step 1 of the exit step; otherwise, proceed to Step 9.

9. Set

$$
\hat{S}(t)=S(D) \quad \text { for } \quad t \in\left[T_{t a d}, T_{t a d}+T_{m i n}^{l o}\right]
$$


and advance the low temperature simulation clock by $T_{m i n}^{l o}$ :

$$
T_{t a d}=T_{t a d}+T_{m i n}^{l o}
$$

10. Let

$$
\left.X_{T_{t a d}}^{l o} \sim\left[\rho^{l o}\left(\partial D_{I_{m i n}^{l o}}\right)\right]^{-1} \rho^{l o}\right|_{\partial D_{I_{m i n}^{l o}}} .
$$

Then go back to the decorrelation step, the basin $D$ now being the one obtained by exiting through $X_{T_{t a d}}^{\text {lo }}$.

Below we comment in more detail on idealized TAD.

- The quasistationary distribution in $D:$ (I1) and (A1).

In idealized TAD, the convergence to local equilibrium (see (M1) and (M2) above) is assumed to be reached, and this is made precise using the QSD $\nu$. In particular, we start the high temperature exit search exactly at the QSD $\nu^{h i}$; see (I1). We will also assume the low temperature dynamics reaches $\nu^{l o}$ at the end of the decorrelation step:

(A1) After the decorrelation step of idealized TAD, the low temperature dynamics is distributed according to the QSD in $D: X_{T_{t a d}}^{l o} \sim \nu^{l o}$.

This will be crucial for extrapolating the exit event at low temperature. Assumption (A1) is justified by the fact that the law of $X_{t}^{\text {lo }}$ in the decorrelation step approaches $\nu^{l o}$ exponentially fast in $T_{\text {corr }}$; see [15, 26, for details. We also refer to [15, 26, for a presentation of algorithms which can be used to sample the QSD.

- The exit position: (I2).

To get exact metastable dynamics, we have to assume that the way the dynamics leaves $D$ near a given saddle point $x_{i}$ does not affect the metastable dynamics in the decorrelation step; see (I2). This can be justified in the small temperature regime by using Theorem 3.2 and some exponential decay results on the normal derivative of the QSD away from saddle points. Indeed, the conditional probability that, given the dynamics leaves through $\partial D_{i}$, it leaves outside a neighborhood of $x_{i}$ is of order $e^{-c \beta}$ as $\beta \rightarrow \infty$ (for a constant $c>0$ ); see [13, 16].

\section{- Replacing the Arrhenius law extrapolation rule: (I3).}

In idealized TAD, we replace the extrapolation formula (2.1) based on the Arrhenius law by the idealized formulas (3.7)- (3.8); see (I3). This is a severe modification, since it makes the algorithm impractical. In particular the quantities $\lambda^{l o}$ and $p_{i}^{l o}$ are not known: if they were, it would be very easy to simulate the exit event from $D$; see Corollary 3.3 above.

It is the aim of Section 4 below to explain how the small temperature assumption is used to get practical estimates of the ratios $\Theta_{i}$. For simplicity we perform this small temperature analysis in one dimension. We will show that $\Theta_{i}$ is indeed close to the formula $\exp \left[-\left(\beta^{h i}-\beta^{l o}\right)\left(V\left(x_{i}\right)-V\left(x_{0}\right)\right)\right]$ used in the original and modified TAD; compare (3.10) with (2.1) and (2.10). We expect the same relation to be true in higher dimensions under appropriate conditions; this will be the subject of another paper.

In the analysis below, we need idealizations (I1) and (I3) to exactly replicate the law of the low temperature exit time and exit region in the exit step; see Theorem 3.7 below. With (I1) and (I3), the inferred low temperature exit events are statistically 
exact. This is based in particular on (A1), namely the fact that the low temperature process is distributed according to $\nu^{l o}$ at the end of the decorrelation step. In addition, after an exit event, the dynamics in the next decorrelation step depends on the exact exit point in $\partial D_{i}$ : this is why we also need (I2) to get exact metastable dynamics; see Theorem 3.6 below.

3.3. Idealized TAD is exact. The aim of this section is to prove the following result:

TheOREM 3.6. Let $X_{t}^{\text {lo }}$ evolve according to (1.1) at $\beta=\beta^{\text {lo }}$. Let $\hat{S}(t)$ be the metastable dynamics produced by Algorithm 3.5 (idealized TAD), assuming (A1), and let idealized TAD have the same initial condition as $X_{t}^{l o}$. Then:

$$
\hat{S}(t)_{t \geq 0} \sim S\left(X_{t}^{l o}\right)_{t \geq 0}
$$

that is, the metastable dynamics produced by idealized TAD has the same law as the (exact) low temperature metastable dynamics.

Due to Corollary 3.3. (A1), (I2), and the fact that the low temperature dynamics is simulated exactly during the decorrelation step, it suffices to prove that the exit step of idealized TAD is exact in the following sense:

TheOrem 3.7. Let $X_{t}^{\text {lo }}$ evolve according to (1.1) at $\beta=\beta^{\text {lo }}$ with $X_{t}^{\text {lo }}$ initially distributed according to the $Q S D$ in $D: X_{0}^{l o} \sim \nu^{l o}$. Let $\tau=\inf \left\{t>0: X_{t}^{l o} \notin D\right\}$ and $I$ be the discrete random variable defined by: for $i=1, \ldots, k$,

$$
I=i \text { if and only if } X_{\tau}^{l o} \in \partial D_{i} .
$$

Let $T_{\text {min }}^{l o}$ and $I_{\text {min }}^{l o}$ be the random variables produced by the exit step of idealized TAD. Then, $\left(T_{\text {min }}^{l o}, I_{\text {min }}^{l o}\right)$ has the same probability law as $(\tau, I)$ :

$$
\left(T_{\text {min }}^{l o}, I_{\text {min }}^{l o}\right) \sim(\tau, I) .
$$

The proof of Theorem 3.7 will use (I1) and (I3) in particular. The theorem shows that the exit event from $D$ produced by idealized TAD is exact in law compared to the exit event that would have occurred at low temperature: the random variable $\left(T_{m i n}^{l o}, I_{m i n}^{l o}\right)$ associated with idealized TAD has the same law as the first exit time and location (from $D$ ) of a dynamics $\left(X_{t}^{l o}\right)_{t \geq 0}$ obeying (1.1) with $\beta=\beta^{l o}$ and $X_{0}^{l o} \sim \nu^{l o}$.

To begin, we provide a simple lemma which shows that we can assume $T_{\text {stop }} \equiv \infty$ without loss of generality. We need this result in order to properly define all the random variables $T_{i}^{h i}$, for $i=1, \ldots, k$, where we recall $k$ denotes the number of saddle points of $V$ on $\partial D$.

Lemma 3.8. Consider the exit step of the idealized TAD, and modify Step 8 as follows:

8. Go back to Step 1 of the exit step.

Thus we loop between Step 1 and Step 8 of the exit step for infinite time, regardless of the values of $T_{\text {sim }}$ and $T_{\text {stop. }}$. Then, $\left(T_{\text {min }}^{l o}, I_{\text {min }}^{l o}\right)$ remains constant for all times $T_{\text {sim }}>T_{\text {stop }}$.

Proof. We want to show that without ever advancing to Step 10, the exit step of idealized TAD produces the same random variable $\left(T_{\text {min }}^{l o}, I_{\text {min }}^{l o}\right)$ as soon as $T_{\text {sim }}>$ $T_{\text {stop. }}$. To see this, note that if $T_{i}^{l o}<T_{m i n}^{l o}$, then from (3.10),

$$
T_{i}^{l o}=T_{i}^{h i} \frac{\lambda^{h i} p_{i}^{h i}}{\lambda^{l o} p_{i}^{l o}}<T_{m i n}^{l o}
$$


and so, comparing with (3.11),

$$
T_{i}^{h i}<T_{m i n}^{l o} \frac{\lambda^{l o} p_{i}^{l o}}{\lambda^{h i} p_{i}^{h i}} \leq \frac{T_{m i n}^{l o}}{C}=T_{\text {stop }} .
$$

Thus, if $T_{\text {sim }}>T_{\text {stop }}$, any escape event will lead to an extrapolated time $T_{i}^{l o}$ which will be larger than $T_{m i n}^{l o}$, and thus will not change the value of $T_{m i n}^{l o}$ anymore.

Let us now identify the laws of the random variables $\left(T_{i}^{h i}\right)_{1 \leq i \leq l}$ produced by idealized TAD.

Proposition 3.9. Consider idealized TAD in the setting of Lemma 3.8, so that all the $T_{i}^{\text {hi }}$ are defined, $i=1,2, \ldots, k$.

Let $\left(\tau^{(j)}, I^{(j)}\right)_{j>1}$ be independent and identically distributed random variables such that $\tau^{(j)}$ is independent from $I^{(j)}, \tau^{(j)} \sim \mathcal{E}\left(\lambda^{h i}\right)$ and for $i=1, \ldots, k, I^{(j)}$ is a discrete random variable with law

$$
\mathbb{P}\left(I^{(j)}=i\right)=p_{i}^{h i}
$$

For $i=1, \ldots, k$ define

$$
N_{i}^{h i}=\min \left\{j: I^{(j)}=i\right\} .
$$

Then we have the following equality in law:

$$
\left(T_{1}^{h i}, \ldots, T_{k}^{h i}\right) \sim\left(\sum_{j=1}^{N_{1}^{h i}} \tau^{(j)}, \ldots, \sum_{j=1}^{N_{k}^{h i}} \tau^{(j)}\right) .
$$

Moreover, (i) $T_{i}^{h i} \sim \mathcal{E}\left(\lambda^{h i} p_{i}^{h i}\right)$ and (ii) $T_{1}^{h i}, T_{2}^{h i}, \ldots, T_{k}^{h i}$ are independent.

Proof. The equality (3.13) follows from Corollary 3.3. since in the exit step of idealized TAD, the dynamics restarts from the QSD $\nu^{\text {hi }}$ after each escape event.

Let us now consider the statement $(i)$. Observe that the moment generating function of an exponential random variable $\tau$ with parameter $\lambda$ is: for $s<\lambda$,

$$
\mathbb{E}[\exp (s \tau)]=\int_{0}^{\infty} e^{s t} \lambda e^{-\lambda t} d t=\frac{\lambda}{\lambda-s} .
$$

So, dropping the superscript $h i$ for ease of notation, we have: for $i \in\{1, \ldots, k\}$, and for $s<\lambda p_{i}$,

$$
\begin{aligned}
\mathbb{E}\left[\exp \left(s T_{i}\right)\right] & =\sum_{m=1}^{\infty} \mathbb{E}\left[\exp \left(s T_{i}\right) \mid N_{i}=m\right] \mathbb{P}\left(N_{i}=m\right) \\
& =\sum_{m=1}^{\infty} \mathbb{E}\left[\exp \left(s \sum_{j=1}^{m} \tau^{(j)}\right)\right]\left(1-p_{i}\right)^{m-1} p_{i} \\
& =\sum_{m=1}^{\infty} \mathbb{E}\left[\exp \left(s \tau^{(1)}\right)\right]^{m}\left(1-p_{i}\right)^{m-1} p_{i} \\
& =\frac{\lambda p_{i}}{\lambda-s} \sum_{m=1}^{\infty}\left(\frac{\lambda\left(1-p_{i}\right)}{\lambda-s}\right)^{m-1} \\
& =\frac{\lambda p_{i}}{\lambda p_{i}-s} .
\end{aligned}
$$


This shows $T_{i}^{h i} \sim \mathcal{E}\left(\lambda^{h i} p_{i}^{h i}\right)$.

Before turning to the proof of the statement (ii) in Proposition 3.9, we need the following technical lemma:

Lemma 3.10. Let $a_{1}, a_{2}, \ldots, a_{n}$ be positive real numbers, and let $S_{n}$ be the symmetric group on $\{1,2, \ldots, n\}$. Then

$$
\sum_{\sigma \in S_{n}} \prod_{i=1}^{n}\left(\sum_{j=i}^{n} a_{\sigma(j)}\right)^{-1}=\prod_{i=1}^{n} a_{i}^{-1} .
$$

Proof. Note that (3.14) is of course true for $n=1$. Assume it is true for $n-1$, and let

$$
S_{n}^{(k)}=\left\{\sigma \in S_{n}: \sigma(1)=k\right\}
$$

Then

$$
\begin{aligned}
\sum_{\sigma \in S_{n}} \prod_{i=1}^{n}\left(\sum_{j=i}^{n} a_{\sigma(j)}\right)^{-1} & =\left(\sum_{i=1}^{n} a_{i}\right)^{-1} \sum_{\sigma \in S_{n}} \prod_{i=2}^{n}\left(\sum_{j=i}^{n} a_{\sigma(j)}\right)^{-1} \\
& =\left(\sum_{i=1}^{n} a_{i}\right)^{-1} \sum_{k=1}^{n} \sum_{\sigma \in S_{n}^{(k)}} \prod_{i=2}^{n}\left(\sum_{j=i}^{n} a_{\sigma(j)}\right)^{-1} \\
& =\left(\sum_{i=1}^{n} a_{i}\right)^{-1} \sum_{\substack{k=1 \\
k}}^{n} \prod_{\substack{j=1 \\
j \neq k}}^{-1} a_{j}^{n} \\
& =\prod_{i=1}^{n} a_{i}^{-1} .
\end{aligned}
$$

By induction (3.14) is valid for all $n$.

We are now in position to prove statement (ii) of Proposition 3.9.

Proof. [Proof of Proposition 3.9 part (ii)] In this proof, we drop the superscript $h i$ for ease of notation. To show that the $T_{i}$ 's are independent, it suffices to show that for $s_{1}, \ldots, s_{k}$ in a neighborhood of zero we have

$$
\mathbb{E}\left[\exp \left(\sum_{i=1}^{k} s_{i} T_{i}\right)\right]=\prod_{i=1}^{k} \mathbb{E}\left[\exp \left(s_{i} T_{i}\right)\right] .
$$

We saw in the proof of part $(i)$ that: for $s_{i}<\lambda p_{i}$,

$$
\mathbb{E}\left[\exp \left(s_{i} T_{i}\right)\right]=\frac{\lambda p_{i}}{\lambda p_{i}-s_{i}}
$$

Consider then the left-hand-side of (3.15). We start by a preliminary computation. 
Let $m_{0}=0, m_{1}=1$, and $s_{i}<\lambda p_{i}$ for $i=1, \ldots, k$. Then

$$
\begin{aligned}
& \sum_{1<m_{2}<m_{3} \ldots<m_{k}} \mathbb{E}\left[\exp \left(\sum_{i=1}^{k} s_{i} T_{i}\right) \mid \cap_{i=1}^{k}\left\{N_{i}=m_{i}\right\}\right] \mathbb{P}\left(\cap_{i=1}^{k}\left\{N_{i}=m_{i}\right\}\right) \\
& =\sum_{1<m_{2}<m_{3} \ldots<m_{k}} \mathbb{E}\left[\exp \left(\sum_{i=1}^{k}\left(s_{i} \sum_{j=1}^{m_{i}} \tau^{(j)}\right)\right)\right] p_{1} \prod_{i=2}^{k} p_{i}\left(1-\sum_{j=i}^{k} p_{j}\right)^{m_{i}-m_{i-1}-1} \\
& =p_{1} \sum_{1<m_{2}<m_{3} \ldots<m_{k}} \prod_{i=1}^{k} \mathbb{E}\left[\exp \left(\left(\sum_{j=i}^{k} s_{j}\right) \sum_{j=m_{i-1}+1}^{m_{i}} \tau^{(j)}\right)\right] \prod_{i=2}^{k} p_{i}\left(1-\sum_{j=i}^{k} p_{j}\right)^{m_{i}-m_{i-1}-1} \\
& =p_{1} \sum_{1<m_{2}<m_{3} \ldots<m_{k}} \prod_{i=1}^{k} \mathbb{E}\left[\exp \left(\tau^{(1)} \sum_{j=i}^{k} s_{j}\right)\right]^{m_{i}-m_{i-1}} \prod_{i=2}^{k} p_{i}\left(1-\sum_{j=i}^{k} p_{j}\right)^{m_{i}-m_{i-1}-1} \\
& =\left(\frac{\lambda p_{1}}{\lambda-\sum_{j=1}^{k} s_{j}}\right) \sum_{1<m_{2}<m_{3} \ldots<m_{k}} \prod_{i=2}^{k} p_{i}\left(\frac{\lambda}{\lambda-\sum_{j=i}^{k} s_{j}}\right)\left(\frac{\lambda\left(1-\sum_{j=i}^{k} p_{j}\right)}{\lambda-\sum_{j=i}^{k} s_{j}}\right)^{m_{i}-m_{i-1}-1} \\
& =\left(\frac{\lambda p_{1}}{\lambda-\sum_{j=1}^{k} s_{j}}\right) \prod_{i=2}^{k} p_{i}\left(\frac{\lambda}{\lambda-\sum_{j=i}^{k} s_{j}}\right)\left(1-\frac{\lambda\left(1-\sum_{j=i}^{k} p_{j}\right)}{\lambda-\sum_{j=i}^{k} s_{j}}\right)^{-1} \\
& =\left(\frac{\lambda p_{1}}{\lambda-\sum_{j=1}^{k} s_{j}}\right) \prod_{i=2}^{k} \lambda p_{i}\left(\sum_{j=i}^{k} \lambda p_{j}-s_{j}\right)^{-1} \\
& =\prod_{i=1}^{k} \lambda p_{i}\left(\sum_{j=i}^{k} \lambda p_{j}-s_{j}\right)^{-1} \text {. }
\end{aligned}
$$

From (3.17) observe that

$$
\begin{aligned}
\mathbb{E}\left[\exp \left(\sum_{i=1}^{k} s_{i} T_{i}\right)\right] & =\sum_{\sigma \in S_{k}} \prod_{i=1}^{k} \lambda p_{\sigma(i)}\left(\sum_{j=i}^{k} \lambda p_{\sigma(j)}-s_{\sigma(j)}\right)^{-1} \\
& =\left(\prod_{i=1}^{k} \lambda p_{i}\right) \sum_{\sigma \in S_{k}} \prod_{i=1}^{k}\left(\sum_{j=i}^{k} \lambda p_{\sigma(j)}-s_{\sigma(j)}\right)^{-1} \\
& =\prod_{i=1}^{k} \frac{\lambda p_{i}}{\lambda p_{i}-s_{i}}
\end{aligned}
$$

where in the last step we have used Lemma 3.10. Comparing (3.15) with (3.16) and (3.18), we are done.

To complete the proof of Theorem 3.7, we finally need the following Lemma.

Lemma 3.11. Let $T_{1}, \ldots, T_{k}$ be independent random variables such that $T_{i} \sim$ $\mathcal{E}\left(\lambda p_{i}\right)$, with $\lambda>0, p_{i}>0$ and $\sum_{j=1}^{k} p_{i}=1$. Set

$$
T=\min _{i} T_{i} \quad \text { and } \quad I=\arg \min _{i} T_{i} .
$$


Then: (i) $T \sim \mathcal{E}(\lambda)$, (ii) $\mathbb{P}(I=i)=p_{i}$, and (iii) $T$ and $I$ are independent.

Proof. Since the $T_{i}$ 's are assumed to be independent, it is well known that $T=$ $T_{I}=\min _{i} T_{i}$ is an exponential random variable with parameter $\sum_{i} \lambda p_{i}=\lambda$. This proves $(i)$. Turning to $(i i)$ and $(i i i)$, note that $\min _{j \neq i} T_{j}$ is an exponential random variable independent of $T_{i}$ with parameter

$$
\sum_{j \neq i} \lambda p_{j}=\lambda\left(1-p_{i}\right)
$$

Thus,

$$
\begin{aligned}
\mathbb{P}\left(I=i, T_{I} \geq t\right) & =\mathbb{P}\left(t \leq T_{i} \leq \min _{j \neq i} T_{j}\right) \\
& =\int_{t}^{\infty} \int_{s}^{\infty} \lambda p_{i} e^{-\lambda p_{i} s} \lambda\left(1-p_{i}\right) e^{-\lambda\left(1-p_{i}\right) r} d r d s \\
& =\int_{t}^{\infty} \lambda p_{i} e^{-\lambda s} d s \\
& =p_{i} \mathbb{P}\left(T_{I} \geq t\right) .
\end{aligned}
$$

Setting $t=0$ we obtain $\mathbb{P}(I=i)=p_{i}$, which proves (ii). Now (iii) follows from (3.19).

口

We are now in position to prove Theorem 3.7.

Proof. [Proof of Theorem 3.7] First, by Lemma 3.8, we can assume that $T_{\text {stop }}=$ $\infty$ so that all the $T_{i}^{h i}$ 's are well defined, for $i=1, \ldots, k$. Then Proposition 3.9 implies that the $T_{i}^{h i}$ s are independent exponential random variables with parameters $\lambda^{h i} p_{i}^{h i}$. So by (3.10), the $T_{i}^{l o}$ 's are independent exponential random variables with parameters $\lambda^{l o} p_{i}^{l o}$. Now by applying Lemma 3.11 to the $T_{i}^{l o}$ 's, we get $T_{\min }^{l o} \sim \mathcal{E}\left(\lambda^{l o}\right)$, $\mathbb{P}\left(I_{m i n}^{l o}=i\right)=p_{i}^{l o}$, and $T_{m i n}^{l o}$ is independent of $I_{m i n}^{l o}$. Referring to Corollary 3.3, we are done.

REMARK 3.12. Observe that the proof of Theorem 3.7 does not use (I2), which is needed only to obtain correct metastable dynamics by iterating the exit step. Also, notice that we did not use the fact that $D$ is the basin of attraction of a local minimum of $V$ or that each set $\partial D_{i}$ in the partition of $\partial D$ is associated to a saddle point $x_{i}$ for the moment. The latter assumption is crucial in the next section, in which we obtain computable estimates of the ratios $\Theta_{i}, i=1, \ldots, k$; this will also require an assumption of large $\beta$ which was not needed for Theorem 3.7.

4. Estimates for the $\Theta_{i}$ 's at low temperature in one dimension. In the last section we showed that modified TAD (Algorithm 2.5) is exact with the idealizations (I1)-(I3) and the assumption (A1); see idealized TAD (Algorithm 3.5). In this section we justify (I3). In particular, we show in Theorem 4.1 below how the ratios $\Theta_{i}$ (see (3.7) ) can be approximated by explicit practical formulas in one dimension. Compared to Theorem 3.7, the proof of Theorem 4.1 will require the additional assumption that temperature is sufficiently small.

4.1. Statement of the main result. We recall that the ratios $\Theta_{i}, i=1, \ldots, k$ are unknown in practice. In TAD these ratios are approximated using the Arrhenius law. The main result of this section, Theorem 4.1 gives precise asymptotics for $\Theta_{i}$ as $\beta^{h i}, \beta^{l o} \rightarrow \infty$. In particular, we show that $\Theta_{i}$ converges to $\exp \left[-\left(\beta^{h i}-\beta^{l o}\right)\left(V\left(x_{i}\right)-\right.\right.$ $\left.\left.V\left(x_{0}\right)\right)\right]$.

Throughout this section we assume that we are in a one dimensional setting. Moreover, we assume that $D$ is the basin of attraction of the gradient dynamics 


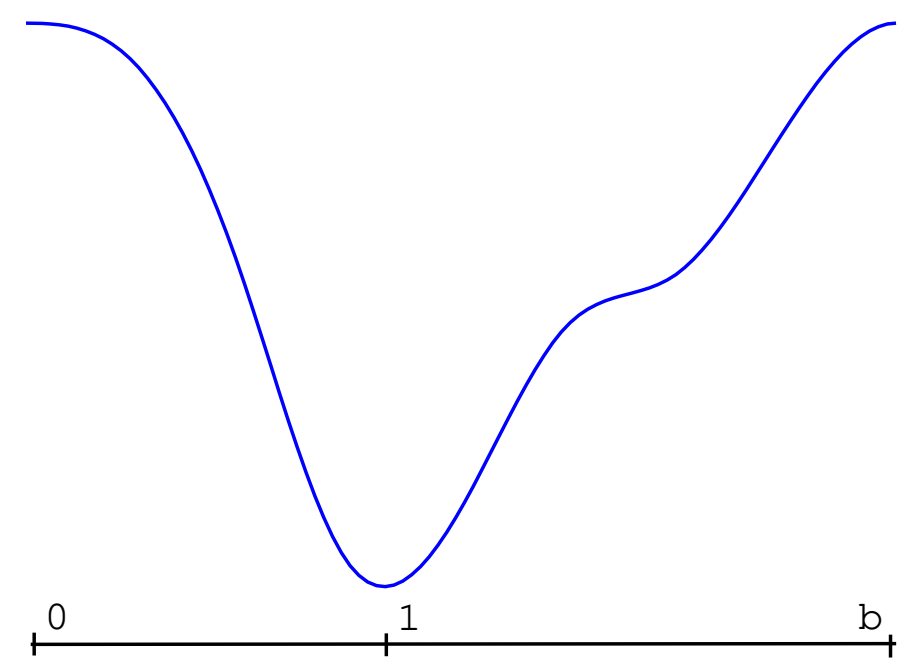

FIG. 4.1. A function $V: D \rightarrow \mathbb{R}$ satisfying (B1)-(B3).

$d y / d t=-V^{\prime}(y)$ associated to a local minimum of $V$ (this is what is done in practice by A.F. Voter and co-workers). Finally, the potential $V$ is assumed to be a Morse function, which means that the critical points of $V$ are non-degenerate. Under these assumptions, we may assume without additional loss of generality that (see Figure 4.1):

(B1) $D=(0, b)$, with $b>1, V(0)=0$, and $V^{\prime}(x) \neq 0$ for $x \notin\{0,1, b\}$,

(B2) $V^{\prime}(0)=0=V^{\prime}(b)$ and $V^{\prime \prime}(0)<0, V^{\prime \prime}(b)<0$,

(B3) $V^{\prime}(1)=0$ and $V^{\prime \prime}(1)>0$.

We also normalize $u$ (see (3.1)) so that

(B4) $u(1)=1$.

In particular, the location of the minimum of $V$ and the value of $V$ at 0 are chosen for notational convenience and without loss of generality. In the following, we write $\{0\}=\partial D_{1}$ and $\{b\}=\partial D_{2}$.

We will prove the following:

THEOREM 4.1. Under the assumptions stated above, we have the formula: for $i=1,2$,

$$
\Theta_{i}=\frac{\lambda^{h i} p_{i}^{h i}}{\lambda^{l o} p_{i}^{l o}}=e^{-\left(\beta^{h i}-\beta^{l o}\right)\left(V\left(x_{i}\right)-V\left(x_{0}\right)\right)}\left(1+O\left(\frac{1}{\beta^{h i}}-\frac{1}{\beta^{l o}}\right)\right)
$$

as $\beta^{h i}, \beta^{l o} \rightarrow \infty, \beta^{l o} / \beta^{h i}=r$ where $x_{1}=0, x_{2}=b$ and $x_{0}=1$, and $r>0$ is constant.

The ratios $\frac{\lambda^{h i} p_{i}^{h i}}{\lambda^{l o} p_{i}^{l o}}$ involve integrals of the form $\int_{D} e^{-\beta V(x)} u(x) d x$ at high and low temperature. We will use Laplace expansions to analyze the integrals, but since $u$ depends on $\beta$, extra care must be taken in the analysis.

4.2. Proof of Theorem 4.1, In all what follows, $(u,-\lambda)$ denotes the principal eigenvector/eigenvalue pair of $L$ with homogeneous Dirichlet boundary conditions; see (3.1). We are interested in how the pair $(u,-\lambda)$ varies in the small temperature regime $\beta \rightarrow \infty$. 
Throughout this section, we write $c$ to denote a positive constant, the value of which may change without being explicitly noted. To begin, we will need some asymptotics for $\lambda$ and $u$, Lemma 4.2 and Lemma 4.3 below. The contents of both lemmas are found in or implied by [6, 9], and [10] (see also [8] and [7) in the case where $V^{\prime} \cdot n>0$ on $\partial D$, with $n$ the normal to $\partial D$ (in our setting $n=1$ on $\partial D_{2}$ and $n=-1$ on $\left.\partial D_{1}\right)$. Here, we consider the case of characteristic boundary, where from (B2) $V^{\prime} \cdot n=0$ on $\partial D$, so we adapt the classical results to this case.

Lemma 4.2. There exists $c>0$ such that

$$
\lambda=O\left(e^{-c \beta}\right) \quad \text { as } \beta \rightarrow \infty .
$$

Proof. Let $D^{\prime} \subset D$ be a domain containing 1 such that $\overline{D^{\prime}} \subset D$, and let $\left(u^{\prime},-\lambda^{\prime}\right)$ the principal eigenvector/eigenvalue pair for $L$ on $D^{\prime}$ with homogeneous Dirichlet boundary conditions on $\partial D^{\prime}$. Recall that $\lambda$ is given by the Rayleigh formula

$$
\lambda=\inf _{f \in H_{V}^{1}(D)} \frac{\beta^{-1} \int_{D}|\nabla f(x)|^{2} e^{-\beta V(x)} d x}{\int_{D} f(x)^{2} e^{-\beta V(x)} d x}
$$

where $H_{V}^{1}(D)$ is the space of functions vanishing on $\mathbb{R} \backslash D$ such that

$$
\int_{D}\left(|\nabla f(x)|^{2}+f(x)^{2}\right) e^{-\beta V(x)} d x<\infty,
$$

and similarly for $\lambda^{\prime}$. Since every function vanishing on $\mathbb{R} \backslash D^{\prime}$ also vanishes on $\mathbb{R} \backslash D$, we have

$$
\lambda \leq \lambda^{\prime}
$$

Now let $X_{t}^{1}$ obey (1.1) with $X_{0}^{1}=1$, and define $\tau^{\prime}=\inf \left\{t>0: X_{t}^{1} \notin D^{\prime}\right\}$. Since $D^{\prime}$ is a sub-basin of attraction such that $V^{\prime}$ points outward on $\partial D^{\prime}$, we can use the following classical results (see e.g. Lemmas $3-4$ of $[\underline{6}$ ):

$$
\lim _{\beta \rightarrow \infty} \beta^{-1} \log \mathbb{E}\left[1 / \lambda^{\prime}\right]=\lim _{\beta \rightarrow \infty} \beta^{-1} \log \mathbb{E}\left[\tau^{\prime}\right]=\inf _{z \in \partial D^{\prime}} \inf _{t>0} I_{z, t}
$$

where, by definition,

$$
\begin{aligned}
& I_{z, t}=\inf _{f \in H_{1}^{z}[0, t]} \frac{1}{4} \int_{0}^{t}\left|\dot{f}(s)+V^{\prime}(f(s))\right|^{2} d s \\
& H_{1}^{z}[0, t]=\left\{f: \exists \dot{f} \in L^{2}[0, t] \text { s.t. } f(t)=z, \forall s \in[0, t], f(s)=1+\int_{0}^{s} \dot{f}(r) d r\right\} .
\end{aligned}
$$

Observe that for any $t>0$ and $f \in H_{1}^{z}[0, t]$ we have

$$
\begin{aligned}
& \frac{1}{4} \int_{0}^{t}\left|\dot{f}(s)+V^{\prime}(f(s))\right|^{2} d s \\
& =\frac{1}{4} \int_{0}^{t}\left|\dot{f}(s)-V^{\prime}(f(s))\right|^{2} d s+\int_{0}^{t} \dot{f}(s) V^{\prime}(f(s)) d s \\
& \geq V(z)-V(1) .
\end{aligned}
$$


Since $\partial D^{\prime}$ is disjoint from 1 we can conclude that for $z \in \partial D^{\prime}, I_{z, t} \geq c>0$ uniformly in $t>0$, for a positive constant $c$. Thus,

$$
\lim _{\beta \rightarrow \infty} \beta^{-1} \log \mathbb{E}\left[\tau^{\prime}\right] \geq c>0
$$

which, combined with (4.3) and (4.4), implies the result.

Next we need the following regularity result for $u$ :

LEMMA 4.3. The function $u$ is uniformly bounded in $\beta$, that is,

$$
\|u\|_{\infty}=O(1) \quad \text { as } \beta \rightarrow \infty,
$$

where $\|\cdot\|_{\infty}$ is the $L^{\infty}$ norm on $C[0, b]$.

Proof. Define $f(t, x)=u(x) e^{\lambda t}$ and set

$$
\tau^{x}=\inf \left\{t>0: X_{t}^{x} \notin(0,1)\right\}
$$

where $X_{t}^{x}$ obeys (1.1) with $X_{0}^{x}=x$. Fix $T>0$. By Itō's lemma, for $t \in\left[0, T \wedge \tau^{x}\right]$ we have

$$
\begin{aligned}
f\left(t, X_{t}^{x}\right) & =u(x)+\lambda \int_{0}^{t} u\left(X_{s}^{x}\right) e^{\lambda s} d s+\int_{0}^{t} L u\left(X_{s}^{x}\right) e^{\lambda s} d s+\sqrt{2 \beta^{-1}} \int_{0}^{t} u^{\prime}\left(X_{s}^{x}\right) d W_{s} \\
& =u(x)+\sqrt{2 \beta^{-1}} \int_{0}^{t} u^{\prime}\left(X_{s}^{x}\right) d W_{s} .
\end{aligned}
$$

Setting $t=T \wedge \tau^{x}$ and taking expectations gives

$$
u(x)=\mathbb{E}\left[f\left(T \wedge \tau^{x}, X_{T \wedge \tau^{x}}^{x}\right)\right]=\mathbb{E}\left[e^{\lambda T \wedge \tau^{x}} u\left(X_{T \wedge \tau^{x}}^{x}\right)\right] .
$$

Recall that $u$ is bounded for fixed $\beta$. We show in equations (4.11) below that $\mathbb{E}\left[e^{\lambda \tau^{x}}\right]$ is finite, so we may let $T \rightarrow \infty$ in (4.6) and use the dominated convergence theorem to obtain

$$
u(x)=\mathbb{E}\left[e^{\lambda \tau^{x}} u\left(X_{\tau^{x}}^{x}\right)\right] \leq \mathbb{E}\left[e^{\lambda \tau^{x}}\right]
$$

where we have recalled $u(0)=0$ and, from (B4), $u(1)=1$. The idea is then to compare $\tau^{x}$ to the first hitting time of 1 of a Brownian motion reflected at zero. Define

$$
\sigma^{x}=\inf \left\{t>0: B_{t}^{x} \notin(-1,1)\right\}
$$

where

$$
B_{t}^{x}=x+\sqrt{2 \beta^{-1}} W_{t}
$$

with $W_{t}^{x}$ as in (1.1). Let $\bar{B}_{t}^{x}$ and $\bar{X}_{t}^{x}$ be given by reflecting $B_{t}^{x}$ and $X_{t}^{x}$ at zero. Since $V^{\prime}<0$ on $(0,1)$, it is clear that $\bar{X}_{t}^{x} \geq \bar{B}_{t}^{x}$ for each $x \in(0,1)$ and $t \geq 0$. Thus,

$$
\begin{aligned}
\mathbb{P}\left(\tau^{x} \geq t\right) & \leq \mathbb{P}\left(\inf \left\{s>0: \bar{X}_{s}^{x}=1\right\} \geq t\right) \\
& \leq \mathbb{P}\left(\inf \left\{s>0: \bar{B}_{s}^{x}=1\right\} \geq t\right) \\
& \leq \mathbb{P}\left(\inf \left\{s>0: \bar{B}_{s}^{0}=1\right\} \geq t\right) \\
& =\mathbb{P}\left(\sigma^{0} \geq t\right) .
\end{aligned}
$$


We will bound from above the last line of (4.8). Let $v(t, x)$ solve the heat equation $v_{t}=\beta^{-1} v_{x x}$ with $v(0, x)=1$ for $x \in(-1,1)$ and $v(t, \pm 1)=0$. An elementary analysis shows that

$$
v(t, 0) \leq \frac{4}{\pi} \exp \left(-\beta^{-1} \pi^{2} t / 4\right) .
$$

(The Fourier sine series for $v(t, x-1)$ on $[0,2]$ at $x=1$ is an alternating series, and its first term gives the upper bound above.) We claim that for fixed $t$ and $x \in[0, t]$,

$$
v(t, 0)=\mathbb{P}\left(\sigma^{0} \geq t\right) .
$$

To see this, let $w(s, x)=v(t-s, x)$ and observe that $w_{s}=-\beta^{-1} w_{x x}$, so by Itō's lemma, for $s \in\left[0, t \wedge \sigma^{x}\right]$

$$
\begin{aligned}
w\left(s, B_{s}^{x}\right) & =w(0, x)+\int_{0}^{s}\left(w_{s}+\beta^{-1} w_{x x}\right)\left(r, B_{r}^{x}\right) d r+\sqrt{2 \beta^{-1}} \int_{0}^{s} w_{x}\left(r, B_{x}^{r}\right) d W_{r} \\
& =w(0, x)+\sqrt{2 \beta^{-1}} \int_{0}^{s} w_{x}\left(r, B_{x}^{r}\right) d W_{r} .
\end{aligned}
$$

By taking expectations and setting $s=t \wedge \sigma^{x}$ we obtain

$$
\begin{aligned}
v(t, x)=w(0, x) & =\mathbb{E}\left[w\left(t \wedge \sigma^{x}, B_{t \wedge \sigma^{x}}^{x}\right)\right] \\
& =\mathbb{E}\left[w\left(t, B_{t}^{x}\right) 1_{\left\{t \leq \sigma^{x}\right\}}\right]+\mathbb{E}\left[w\left(\sigma^{x}, B_{\sigma^{x}}^{x}\right) 1_{\left\{t>\sigma^{x}\right\}}\right] \\
& =\mathbb{E}\left[v\left(0, B_{t}^{x}\right) 1_{\left\{t \leq \sigma^{x}\right\}}\right] \\
& =\mathbb{P}\left(\sigma^{x} \geq t\right) .
\end{aligned}
$$

From (4.8), (4.9) and (4.10), for $x \in[0,1)$

$$
\mathbb{P}\left(\tau^{x} \geq t\right) \leq \frac{4}{\pi} \exp \left(-\beta^{-1} \pi^{2} t / 4\right) .
$$

By Lemma 4.2, $\lambda \beta \rightarrow 0$ as $\beta \rightarrow \infty$. So for all sufficiently large $\beta$,

$$
\begin{aligned}
\mathbb{E}\left[e^{\lambda \tau^{x}}\right] & =1+\int_{1}^{\infty} \mathbb{P}\left(e^{\lambda \tau^{x}} \geq t\right) d t \\
& \leq 1+\frac{4}{\pi} \int_{1}^{\infty} t^{-\pi^{2} /(4 \lambda \beta)} d t \\
& =1+\frac{4}{\pi} \frac{4 \lambda \beta}{\pi^{2}-4 \lambda \beta} .
\end{aligned}
$$

Now recalling (4.7),

$$
u(x) \leq \mathbb{E}\left[e^{\lambda \tau^{x}}\right] \leq 1+\frac{4}{\pi} \frac{4 \lambda \beta}{\pi^{2}-4 \lambda \beta} .
$$

Using Lemma 4.2 we see that the right hand side of (4.12) approaches 1 as $\beta \rightarrow \infty$. An analogous argument can be made for $x \in(1, b]$, showing that $u$ is uniformly bounded in $\beta$ as desired.

Next we define a function which will be useful in the analysis of (3.7). For $x \in[0,1]$ let

$$
f(x)=\frac{\int_{0}^{x} e^{\beta V(t)} d t}{\int_{0}^{1} e^{\beta V(t)} d t} .
$$


We compare $u$ and $f$ in the following lemma:

Lemma 4.4. Let $\|\cdot\|_{\infty}$ the $L^{\infty}$ norm on $C[0,1]$. With $f$ defined by (4.13), we have, in the limit $\beta \rightarrow \infty$,

$$
\begin{aligned}
& \|f-u\|_{\infty}=O\left(e^{-c \beta}\right), \\
& \left\|f^{\prime}-u^{\prime}\right\|_{\infty}=O\left(e^{-c \beta}\right) .
\end{aligned}
$$

Proof. Observe that $g=f-u$, defined on $[0,1]$, satisfies

$$
\begin{array}{r}
-V^{\prime}(x) g^{\prime}(x)+\beta^{-1} g^{\prime \prime}(x)=\lambda u(x) \\
g(0)=0, \quad g(1)=0 .
\end{array}
$$

Multiplying by $\beta e^{-\beta V(x)}$ in (4.14) leads to

$$
\frac{d}{d x}\left(e^{-\beta V(x)} g^{\prime}(x)\right)=\beta e^{-\beta V(x)} \lambda u(x)
$$

so that

$$
g^{\prime}(x)=e^{\beta V(x)}\left(\lambda \beta \int_{0}^{x} e^{-\beta V(t)} u(t) d t+C_{\beta}\right) .
$$

Integrating (4.15) and using $g(0)=0$,

$$
g(x)=\lambda \beta \int_{0}^{x}\left(e^{\beta V(t)} \int_{0}^{t} e^{-\beta V(s)} u(s) d s\right) d t+C_{\beta} \int_{0}^{x} e^{\beta V(t)} d t .
$$

Using Lemma 4.3 we have $\|u\|_{\infty} \leq K<\infty$. From (B1) and (B3) we see that $V$ is decreasing on $[0,1]$. So putting $g(1)=0$ in (4.16) we obtain, for all sufficiently large $\beta$,

$$
\begin{aligned}
\left|C_{\beta}\right| & =\lambda \beta\left(\int_{0}^{1} e^{\beta V(t)} d t\right)^{-1} \int_{0}^{1}\left(e^{\beta V(t)} \int_{0}^{t} e^{-\beta V(s)} u(s) d s\right) d t \\
& \leq \lambda \beta\left(\int_{0}^{1} e^{\beta V(t)} d t\right)^{-1} \int_{0}^{1}\left(\int_{0}^{t} u(s) d s\right) d t \\
& \leq \lambda \beta K\left(\int_{0}^{1} e^{\beta V(t)} d t\right)^{-1} \\
& \leq 2 \lambda \beta^{3 / 2} K\left(\frac{-2 V^{\prime \prime}(0)}{\pi}\right)^{1 / 2}
\end{aligned}
$$

where in the last line Laplace's method is used. Using Lemma 4.2, for all sufficiently large $\beta$,

$$
\left|C_{\beta}\right| \leq e^{-c \beta}
$$

From (B1) and (B3) we see that $V$ is nonpositive on $[0,1]$, so from (4.15),

$$
\begin{aligned}
\left|g^{\prime}(x)\right| & \leq \lambda \beta \int_{0}^{x} e^{\beta(V(x)-V(t))} u(t) d t+\left|C_{\beta}\right| e^{\beta V(x)} \\
& \leq \lambda \beta K+e^{-c \beta} .
\end{aligned}
$$


Using Lemma 4.2 again, we get $\left\|g^{\prime}\right\|_{\infty}=O\left(e^{-c \beta}\right)$. As $g(0)=0$ this implies $\|g\|_{\infty}=$ $O\left(e^{-c \beta}\right)$. This completes the proof. $\mathbf{Q}$

REMARK 4.5. A result analogous to Lemma 4.3 holds, with

$$
f(x)=\frac{\int_{x}^{b} e^{\beta V(t)} d t}{\int_{1}^{b} e^{\beta V(t)} d t},
$$

for $x \in[1, b]$.

We are now in position to prove Theorem 4.1 .

Proof. [Proof of Theorem 4.1] It suffices to prove the case $i=1$, so we will look at the endpoint $\partial D_{1}=\{0\}$. From Theorem 3.2 we have

$$
\rho(\{0\})=\frac{\left.\frac{d}{d x}\left(u(x) e^{-\beta V(x)}\right)\right|_{x=0}}{\beta \lambda \int_{D} u(x) e^{-\beta V(x)} d x}
$$

so that

$$
\lambda p_{1}=\frac{e^{-\beta V(0)} u^{\prime}(0)}{\beta \int_{D} u(x) e^{-\beta V(x)} d x} .
$$

Introducing again the superscripts ${ }^{h i}$ and ${ }^{l o}$,

$$
\frac{\lambda^{h i} p_{1}^{h i}}{\lambda^{l o} p_{1}^{l o}}=e^{-\left(\beta^{h i}-\beta^{l o}\right) V(0)} \cdot \frac{\beta^{l o}}{\beta^{h i}} \cdot \frac{u^{h i^{\prime}}(0)}{u^{l o^{\prime}}(0)} \cdot \frac{\int_{D} u^{l o}(x) e^{-\beta^{l o} V(x)} d x}{\int_{D} u^{h i}(x) e^{-\beta^{h i} V(x)} d x}
$$

Dropping the superscripts, recalling the function $f$ from (4.13), and using Lemma4.4, we see that

$$
u^{\prime}(0)=f^{\prime}(0)+O\left(e^{-c \beta}\right) .
$$

Since

$$
f^{\prime}(0)=\left(\int_{0}^{1} e^{\beta V(t)} d t\right)^{-1}=\left(1+k_{1} \beta^{-1}+O\left(\beta^{-2}\right)\right) \sqrt{\beta}\left(\frac{-2 V^{\prime \prime}(0)}{\pi}\right)^{1 / 2}
$$

where $k_{1}$ is a $\beta$-independent constant coming from the second term in the Laplace expansion. Thus

$$
\begin{aligned}
u^{\prime}(0) & =O\left(e^{-c \beta}\right)+\left(1+k_{1} \beta^{-1}+O\left(\beta^{-2}\right)\right) \sqrt{\beta}\left(\frac{-2 V^{\prime \prime}(0)}{\pi}\right)^{1 / 2} \\
& =\left(1+k_{1} \beta^{-1}+O\left(\beta^{-2}\right)\right) \sqrt{\beta}\left(\frac{-2 V^{\prime \prime}(0)}{\pi}\right)^{1 / 2} .
\end{aligned}
$$

This takes care of the third term of the product in 4.20). We now turn to the fourth term. Let $y \in(0,1]$ and note that for $t \in(y, 1]$,

$$
f^{\prime}(t)=e^{\beta V(t)}\left(\int_{0}^{1} e^{\beta V(x)} d x\right)^{-1}=O\left(e^{-c \beta}\right)
$$

where here $c$ depends on $y$. Since $f(1)=1$, for all sufficiently large $\beta$,

$$
|f(t)-1| \leq e^{-c \beta}
$$


for $t \in[y, 1]$ and a different $c$. Also,

$$
\int_{y}^{1} e^{-\beta V(x)} d x=\left(1+k_{2} \beta^{-1}+O\left(\beta^{-2}\right)\right) \sqrt{\beta^{-1}}\left(\frac{\pi}{2 V^{\prime \prime}(1)}\right)^{1 / 2} e^{-\beta V(1)} .
$$

where $k_{2}$ is a $\beta$-independent constant coming from the second term in the Laplace expansion. Thus

$$
\begin{aligned}
\int_{0}^{1} f(x) e^{-\beta V(x)} d x & =O\left(e^{-\beta V(y)}\right)+\int_{y}^{1} f(x) e^{-\beta V(x)} d x \\
& =O\left(e^{-\beta V(y)}\right)+\left(1+O\left(e^{-c \beta}\right)\right) \int_{y}^{1} e^{-\beta V(x)} d x \\
& =O\left(e^{-\beta V(y)}\right)+\left(1+k_{2} \beta^{-1}+O\left(\beta^{-2}\right)\right) \sqrt{\beta^{-1}}\left(\frac{\pi}{2 V^{\prime \prime}(1)}\right)^{1 / 2} e^{-\beta V(1)} \\
& =\left(1+k_{2} \beta^{-1}+O\left(\beta^{-2}\right)\right) \sqrt{\beta^{-1}}\left(\frac{\pi}{2 V^{\prime \prime}(1)}\right)^{1 / 2} e^{-\beta V(1)} .
\end{aligned}
$$

Using (4.23) and Lemma 4.4 again,

$$
\int_{0}^{1} u(x) e^{-\beta V(x)} d x=\left(1+k_{2} \beta^{-1}+O\left(\beta^{-2}\right)\right) \sqrt{\beta^{-1}}\left(\frac{\pi}{2 V^{\prime \prime}(1)}\right)^{1 / 2} e^{-\beta V(1)} .
$$

From Remark 4.5. we can make an identical argument on $[1, b)$ to get

$$
\int_{D} u(x) e^{-\beta V(x)} d x=\left(1+k_{2} \beta^{-1}+O\left(\beta^{-2}\right)\right) \sqrt{\beta^{-1}}\left(\frac{2 \pi}{V^{\prime \prime}(1)}\right)^{1 / 2} e^{-\beta V(1)} .
$$

with a different but still $\beta$-independent $k_{2}$. This takes care of the fourth term in the product in (4.20). Observe that in the limit $\beta^{h i}, \beta^{l o} \rightarrow \infty, \beta^{l o} / \beta^{h i}=r$ we have:

$$
\begin{aligned}
& \frac{1+k_{1}\left(\beta^{h i}\right)^{-1}+O\left(\left(\beta^{h i}\right)^{-2}\right)}{1+k_{1}\left(\beta^{l o}\right)^{-1}+O\left(\left(\beta^{l o}\right)^{-2}\right)}=1+O\left(\frac{1}{\beta^{h i}}-\frac{1}{\beta^{l o}}\right) \\
& \frac{1+k_{2}\left(\beta^{l o}\right)^{-1}+O\left(\left(\beta^{l o}\right)^{-2}\right)}{1+k_{2}\left(\beta^{h i}\right)^{-1}+O\left(\left(\beta^{h i}\right)^{-2}\right)}=1+O\left(\frac{1}{\beta^{h i}}-\frac{1}{\beta^{l o}}\right) .
\end{aligned}
$$

Reintroducing the superscripts ${ }^{h i}$ and ${ }^{l o}$ and using (4.21) and (4.24) in (4.20) now gives

$$
\frac{\lambda^{h i} p_{1}^{h i}}{\lambda^{l o} p_{1}^{l o}}=\left(1+O\left(\frac{1}{\beta^{h i}}-\frac{1}{\beta^{l o}}\right)\right) e^{-\left(\beta^{h i}-\beta^{l o}\right)(V(0)-V(1))}
$$

as desired. $\square$

5. Conclusion. We have presented a mathematical framework for TAD which is valid in any dimension, along with a complete analysis of TAD in one dimension under this framework. This framework uses the notion of quasi-stationary distribution, and is useful in particular to clarify the immediate equilibration assumption (or no-recrossing assumption) which is underlying the original TAD algorithm and to understand the extrapolation rule using the Arrhenius law. We hope to extend this 
justification of the extrapolation rule to high dimensions, using techniques from [16]; the analysis seems likely to be technically detailed.

We hope that our framework for TAD will be useful in cases where the original method is not valid. Indeed, we have shown that TAD can be implemented wherever accurate estimates for the ratios in (3.7) are available. This fact is important for transitions which pass through degenerate saddle points, in which case a pre-exponential factor is needed on the right hand side of (4.1). For example, in one dimension, a simple modification of our analysis shows that if we consider degenerate critical points on $\partial D$, then a factor of the form $\left(\beta^{h i} / \beta^{l o}\right)^{\alpha}$ must be multiplied with the right hand side of (4.1).

Acknowledgments. D. ARISTOFF gratefully acknowledges enlightening discussions with G. Simpson and O. Zeitouni. D. Aristoff and T. Lelièvre acknowledge fruitful input from D. Perez and A.F. Voter. Part of this work was completed while T. LELIÈVRE was an Ordway visiting professor at the University of Minnesota. The work of D. ARISTOFF was supported in part by DOE Award de-sc0002085.

\section{REFERENCES}

[1] X.M. Bai, A. El-Azab, J. Yu, And T.R. Allen, Migration mechanisms of oxygen interstitial clusters in UO2, J. Phys. Condens. Matter., 25(1) (2013), pp. 015003.

[2] N. Berglund, Kramers law: Validity, derivations and generalisations, Markov Processes Relat. Fields, 19 (2013), pp. 459-490.

[3] A.V. Bochenkova And V.E. Bochenkov, HArF in Solid Argon Revisited: Transition from Unstable to Stable Configuration, J. Phys. Chem. A, 113(26) (2009), pp. 7654-7659.

[4] A. Bovier, M. Eckhoff, V. Gayrard, and M. Klein, Metastability in reversible diffusion dynamics I. Sharp asymptotics for capacities and exit times, J. Eur. Math. Soc., 6(4) (2004), pp. 399-424.

[5] M. Cogoni, B.P. Uberuaga, A.F. Voter, and L. Colombo, Diffusion of small self-interstitial clusters in silicon: temperature-accelerated tight-binding molecular dynamics simulations, Phys. Rev. B, 71 (2005), pp. 121203-1-4.

[6] M.V. DAY, On the exponential exit law in the small parameter exit problem, Stochastics, 8 (1983), pp. 297-323.

[7] M.V. DAY, Mathematical Approaches to the Problem of Noise-Induced Exit, Systems and Control: Foundations and Applications, Springer, 1999, pp. 267-287.

[8] A. Dembo and O. Zeitouni, Large deviations techniques and applications, Second ed., Springer Verlag, New York, 1998.

[9] A. Devinatz and A. Friedman, Asymptotic Behavior of the Principal Eigenfunction for a Singularly Perturbed Dirichlet Problem, Indiana Univ. Math. J., 27 (1978), pp. 143-157.

[10] A. Friedman Stochastic differential equations and applications, Vol. 2, Academic Press, New York, 1976.

[11] P. Hänggi, P. Talkner, And M. Borkovec, Reaction-rate theory: fifty years after Kramers, Rev. Mod. Phys., 62(2) (1990), pp. 251-342.

[12] D.J. Harris, M.Y. Lavrentiev, J.H. Harding, N.L. Allan, and J.A. Purton, Novel exchange mechanisms in the surface diffusion of oxides, J. Phys. Condens. Matter, 16 (2004), pp. 187-92.

[13] B. HeLFFER AND F. NIER, Quantitative analysis of metastability in reversible diffusion processes via a Witten complex approach : the case with boundary, Mémoires de la SMF, 105 (2006).

[14] G. Henkelman, B.P. Uberuaga, and H. Jónsson, A climbing image nudged elastic band method for finding saddle points and minimum energy paths, J. Chem. Phys., 113 (2000), pp. 9901-9904.

[15] C. Le Bris, T. Lelièvre, M. Luskin, and D. Perez, A mathematical formulation of the parallel replica dynamics, Monte Carlo Methods Appl., 18 (2012), pp. 119-146.

[16] T. LeLièvre AND F. Nier, Low temperature asymptotics for Quasi-Stationary Distribution in a bounded domain, arXiv:1309.3898.

[17] G. Menz And A. Schlichting, Poincaré and logarithmic Sobolev inequalities by decomposition of the energy landscape, arXiv:1202.1510 
[18] F. Montalenti, M.R. Sørensen, And A.F. Voter, Closing the gap between experiment and theory: crystal growth by temperature accelerated dynamics, Phys. Rev. Lett., 87 (2001), pp. 126101-1-4.

[19] F. Montalenti And A.F. Voter Exploiting past visits or minimum-barrier knowledge to gain further boost in the temperature-accelerated dynamics method, J. Chem. Phys., 116 (2002), pp. 4819-4828.

[20] F. Montalenti, A.F. Voter, and R. Ferrando, Spontaneous atomic shuffle in flat terraces: Ag(100), Phys. Rev. B, 66 (2002), pp. 205404-1-7.

[21] N. Mousseau, L.K. Béland, P. Brommer, J.F. Joly, F. El-Mellouhi, E. MachadoCharry, M.C. Marinica, And P. Pochet, The Activation-Relaxation Technique: ART Nouveau and Kinetic ART, Journal of Atomic, Molecular, and Optical Physics, (2012), pp. 925278 .

[22] F. NIER, Boundary conditions and subelliptic estimates for geometric Kramers-Fokker-Planck operators on manifolds with boundaries, arXiv 1309.5070.

[23] B. ØKSEndal, Stochastic differential equations: an introduction with applications, Springer Verlag, New York, 2003.

[24] C.W. Pao, T.H. Liu, C.C. Chang, and D.J. Srolovitz, Graphene defect polarity dynamics, Carbon, 50(8) (2012), pp. 2870-2876.

[25] D. Perez, B.P. Uberuaga, Y. Shim, J.G. Amar, and A.F. Voter, Accelerated molecular dynamics methods: introduction and recent developments, Annual Reports in Computational Chemistry, 5 (2009), pp. 79-98.

[26] G. Simpson and M. Luskin, Numerical Analysis of Parallel Replica Dynamics, ESAIM: Mathematical Modelling and Numerical Analysis, 47(5) (2013), pp. 1287-1314.

[27] M.R. Sørensen And A.F. Voter, Temperature-accelerated dynamics for simulation of infrequent events, J. Chem. Phys., 112 (2000), pp. 9599-9606.

[28] J.A. Sprague, F. Montalenti, B.P. Uberuaga, J.D. Kress, and A.F. Voter, Simulation of growth of $\mathrm{Cu}$ on $\mathrm{Ag}(001)$ at experimental deposition rates, Phys. Rev. B, 66 (2000), pp. 205415-1-10.

[29] D.G. Tsalikis, N. Lempesis, G.C. Boulougouris, and D.N. Theodorou, Temperature accelerated dynamics in glass forming materials, J. Chem. Phys. B, 114 (2010), pp. 7844-7853.

[30] B.P. Uberuaga, R. Smith, A.R. Cleave, G. Henkelman, R.W. Grimes, A.F. Voter, and K.E. SickAfus, Dynamical simulations of radiation damage and defect mobility in $\mathrm{MgO}$, Phys. Rev. B, 71 (2005), pp. 104102-1.

[31] B.P. Uberuaga, R. Smith, A.R. Cleave, F. Montalenti, G. Henkelman, R.W. Grimes, A.F. Voter, AND K.E. SickAfus, Structure and mobility of defects formed from collision cascades in MgO, Phys. Rev. Lett., 92 (2004), pp. 115505-4.

[32] B.P. Uberuaga, S.M. Valone, M.I. Baskes, And A.F. Voter, Accelerated molecular dynamics study of vacancies in Pu, AIP Conf. Proc., 673 (2003), pp. 213-5.

[33] A.F. Voter, Introduction to the kinetic Monte Carlo method, In Radiation Effects in Solids, Springer, NATO Publishing Unit, Dordrecht, Netherlands, 2006, pp. 1-24.

[34] A.F. Voter, (1998) Parallel replica method for dynamics of infrequent events, Phys. Rev. B, 57 (1998), R13985-88.

[35] A.F. Voter, Private communication. 